\title{
A STUDY OF THE INDUCTANCE OF FOUR-TERMINAL RESISTANCE STANDARDS
}

\author{
By Francis B. Silsbee
}

\section{CONTENTS}

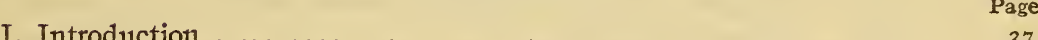

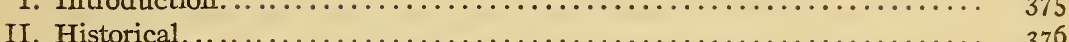

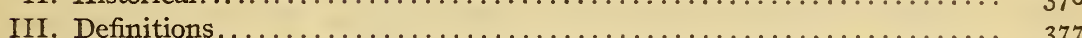



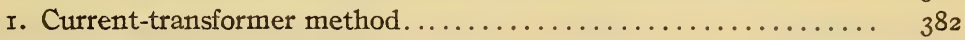

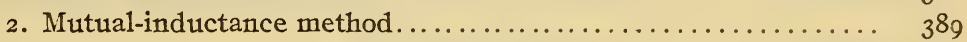

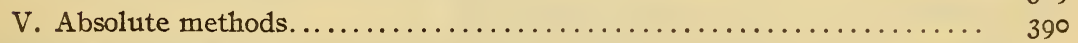

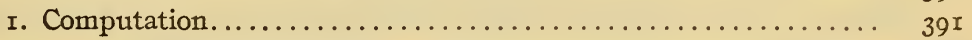



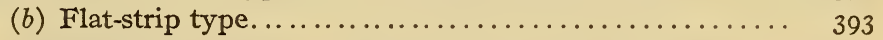

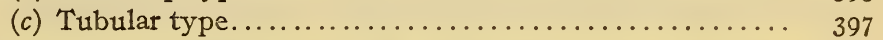

2. Quarter-phase method.......................... 402

3. Change-of-resistance method....................... 403

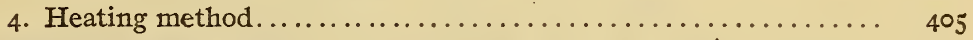

VI. Adjustment of final values................................. 406

VII. Effect of stray fields................................ 408

VIII. Observations on various types of shunt.................... 409

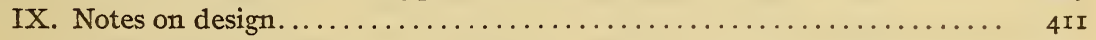

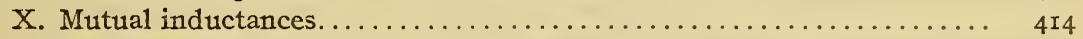

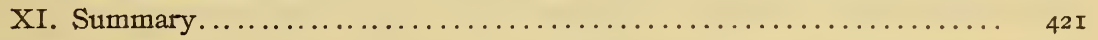

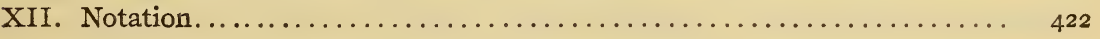

\section{INTRODUCTION}

Potentiometer and bridge methods are coming more and more into use in precise alternating-current measurements, and have created a demand for resistances and mutual inductances of known phase angle. This is particularly true of the present precise methods of testing instrument transformers. ${ }^{1}$

The residual inductance of resistance coils has been pretty thoroughly studied ${ }^{2}$ in the range from I ohm to $10000 \mathrm{ohms}$,

1 As an example of the engineering importance of this work, consider a direct measurement of the power consumed in one of the current limiting reactances used by the New York Edison Co. The 2000-ampere current transformer needed would require the use of a $0.000 \mathrm{I}-\mathrm{ohm}$ resistance standard for its calibration. An error as small as $\mathrm{x} \mathrm{X}_{10^{-9}}$ henry in the assumed inductance of this standard would introduce an error of 33 per cent into the power measurement.

2 Grover and Curtis, this Bulletin, 8, pp. 455, 495 (Reprints No. I.75 and No. 177); Wagner, Elektrotech. Zs. 34, p. 613, 19I3; 36, pp. 606, 621, 1915. 
but comparatively little work has been done above and below this range.

The object of the work described below was to study the range below I ohm. In this range standard resistances are almost invariably of the four-terminal type, and therefore require methods of measurement quite distinct from those applicable to the higher resistances. It was soon evident that the methods of comparing the time constants of two four-terminal resistances were much simpler and more accurate than the determination of the absolute value of any one. The work, therefore, naturally divides itself into two parts-first, the development of a method of comparison, and, second, a study of the various methods for the absolute measurement or computation of the inductance. The latter work has resulted in the establishment at the Bureau of Standards of a series of low resistances whose time constants are known to $0 . \mathrm{I}$ or 0.2 microsecond.

Some of the methods used involved the use of mutual inductances of heavy-current capacity, and considerable time was spent in a study of the phase relations in such apparatus.

\section{HISTORICAL}

In $1906 \mathrm{E}$. and W. H. Wilson ${ }^{3}$ published an account of some measurements on the inductance of shunts using an electrometer method. Since then, however, the subject has been confined to the standardizing laboratories where the need of precise values was first felt. At the National Physical Laboratory in I909 Paterson and Rayner ${ }^{4}$ constructed a series of shunts for use with alternating currents. Using manganin tubes cooled by a vigorous circulation of water they were able to obtain 2.5 volts drop in shunts as low as 0.001 ohm. They kept the time constant of their shunts small by the device suggested by Campbell ${ }^{5}$ of using as potential lead a thin copper sheath just outside the working material. They computed the inductance directly from formulæ given by Russell ${ }^{6}$ and did not attempt a direct measurement.

A few months later Orlich ${ }^{7}$ published a description of the standards in use at the Reichsanstalt. These are of the flatstrip type. The inductances were measured by an electrometer method at 1935 cycles per second. Since this method gave only relative values of the time constants, Orlich constructed as stand-

${ }^{3}$ Electrician, 56, p. $464 ; 1906$.

${ }^{4}$ Inst. Elect. Eng. Journa1, 42, p. 455; 1909.

${ }^{5}$ Electrician, 61, p. 1000; 1908.
${ }^{6}$ Russell, Alt. Currents, 1, p. 53 .

${ }^{7}$ Zeit. für Instrumentenkunde, 29, p. 24x; I909. 
ards two wire rectangles having inductances of about 2 microhenrys each. The measurements on the shunts of lower resistance were made by stepping down through the series.

At the Bureau of Standards Agnew and Fitch ${ }^{8}$ used a dynamometer method analogous electromagnetically to the electrostatic method of Orlich and Wilson. They compared their working shunts with two standards, the inductance of which could be computed from the dimensions.

Sharp and Crawford ${ }^{9}$ in I910 published a description of a series of noninductive shunts constructed along the lines suggested by Drysdale. They were calibrated by stepping down from o.I ohm resistances which were assumed noninductive. The relative measurements were made by a Thomson double bridge, using a dynamometer excited in quadrature with the current in the resistance, as a detector.

\section{DEFINITIONS}

Standard resistances of less than I ohm are usually constructed with four terminals, to which leads may be attached. Two of these (the current terminals) are of comparatively massive construction and are intended to carry the current to be measured, while the other pair (the potential terminals) are smaller and are intended to be connected to some apparatus for measuring difference of potential. This form of construction is used for directcurrent measurements because the value of the resistance is thereby made more independent of the manner in which the current leads are attached and because contact resistance is eliminated. ${ }^{10}$ For alternating-current work this construction has the further advantage that the inductance of the apparatus which would otherwise be extremely variable is thereby made much more definite.

The various factors affecting the inductance of a four-terminal resistance standard (or as we shall for brevity call it "shunt") may be seen from a consideration of Fig. I. If a sinusoidal alternating current $I$ be passed through a piece of resistance material as $a b$, there will be a certain voltage between points $a$ and $b$. This voltage will not, in general, be in phase with the current, but may be resolved into an in-phase and a quadrature component. The ratio of the in-phase component of voltage to the current may

8 This Bulletin, 6, p. 28r; 1909 (Reprint No. I30).

${ }^{9}$ A. I. E. E. Trans., 29, p. I537; I9ro.

${ }^{10}$ For some general relations among four-terminal resistances see Wenner, this Bulletin, 8, p. 559 (Reprint No. I8r), I912; Searle, Electrician, 66, pp. 999, I029; 67, pp. I2, 54, I9гr. 
be taken as a definition of the resistance, and the ratio of the quadrature component of voltage to the current, as the reactance of the piece $a b$. The inductance is, of course, the reactance divided by $2 \pi$ times the frequency. This inductance may be denoted by $L_{\mathrm{ab}}$.

Now, in order that the current may flow through $a b$, the shunt must form part of a closed circuit containing a source of power, and the rest of the circuit will have a certain inductive effect on the voltage between $a$ and $b$. Denoting the mutual inductance between the rest of the circuit and the part $a b$ by $M_{\text {ac }}$, ab we see that the resultant quadrature voltage between $a$ and $b$ will not be

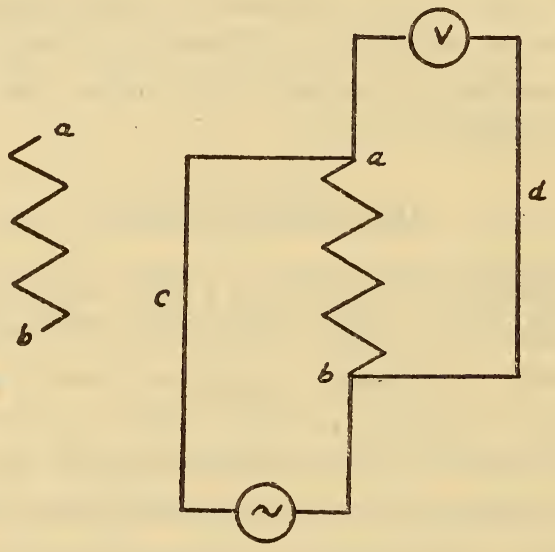

(a)

(b)

Fig. I

$I \omega L_{\mathrm{ab}}$, but $I \omega\left(L_{\mathrm{ab}} \pm M_{\mathrm{ac}}, \mathrm{ab}\right)$. The quantity ( $\left.L_{\mathrm{ab}} \pm M_{\mathrm{ac}}, \mathrm{ab}\right)$ may be called the "inductance of $a b$ with return at $a c$," and denoted by $L_{\mathrm{ab}}$, ac.

Furthermore, in order that the voltage between $a$ and $b$ may be measured, a second closed circuit must be formed as abd containing some arrangement for measuring voltage as $V$. Now there may be mutual inductance between the potential leads, such as $b d$, and the various parts of the current circuit. Let us denote these by $M_{\mathrm{ab}}$, bd and $M_{\mathrm{ac}}$, bd. The quadrature component of the voltage as measured by $V$ will then be not

$$
I \omega L_{\mathrm{ab}} \text {, ac, but } I \omega\left(L_{\mathrm{ab}}, \mathrm{ac} \pm M_{\mathrm{ab}}, \mathrm{bd} \pm M_{\mathrm{ac}}, \mathrm{bd}\right)
$$

or in more detailed form

$$
I \omega\left(L_{\mathrm{ab}} \pm M_{\mathrm{ac}}, \mathrm{ab} \pm M_{\mathrm{ab}}, \mathrm{bd} \pm M_{\mathrm{ac}}, \mathrm{bd}\right)
$$


This quantity depends, in general, on the entire measuring circuit. It is desirable in practice to be able to consider the behavior of the shunt itself as distinct from the rest of the circuit. If the circuits are arranged as in Fig. $2(a)$, we see that the two main circuits acekfb, and abdhvg may each be split up into two circuits each of which is nearly closed. The circuits $I$ and $I V$ are external to the shunt and any mutual inductance between $I$ and $I I I$ or $I I$ and $I V$ may be made very small by proper arrangement of the apparatus and, in any event, may be classed as due to "stray fields" and not as part of the behavior of the shunt. This is also the case with the direct effect of $I V$ upon $I$.

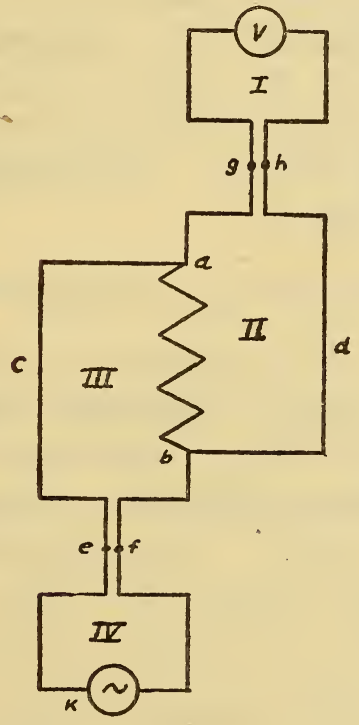

(a)

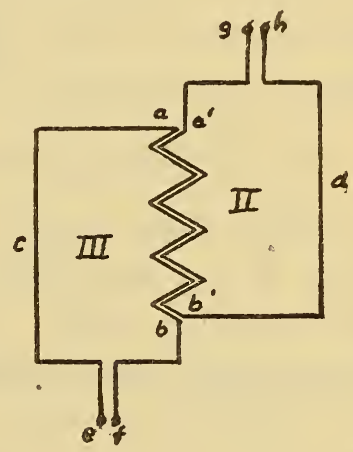

(b)

FIG. 2

The points $g h$ and $e f$, where the division into internal and external circuits occurs, are the terminals of the apparatus. We therefore get as the expression for the inductance of the shunt, the ratio of the quadrature component of the voltage between $g$ and $h$ to $\omega$ times the current flowing between $e$ and $f$. This is the same as

$$
L=L_{\mathrm{ab}} \pm M_{\mathrm{ac}}, \mathrm{ab} \pm M_{\mathrm{ab}}, \mathrm{bd} \pm M_{\mathrm{ac}}, \mathrm{bd}
$$

where the M's must now be considered as including only the mutual effects of circuits inside the terminals. In case the members of each pair of terminals are not close together, the position of the leads attached to them must be specified or the inductance 
will be indefinite. It is, of course, desirable to have the potential terminals as far away as possible from the current terminals, as the liability to error from stray fields is thus reduced.

It is useful in this connection to note that shunts may be divided into two main classes. In type I the working material itself is formed into a closed circuit. With this construction the current leads and the potential leads may be kept close together, and, if so, all the $M$ 's of equation (I) vanish and the inductance becomes that of a closed circuit and is, therefore, always positive. In type II the working material does not form a closed circuit and the inductance may be either positive or negative.

Instead of approaching the problem piecemeal, as was done above, we may consider two circuits $I I$ and $I I I$, as shown in Fig. 2 (b). The quadrature component of the voltage measured by $V$ is $I \omega M_{\mathrm{II}} \mathrm{m}$, where $I$ is the current flowing in circuit $I I$ and $M_{\mathrm{II}, \mathrm{II}}$ is the mutual inductance of the two circuits. This quantity $M_{\text {II, III }}$ may be split up into its parts as follows:

$$
M_{\mathrm{II}, \mathrm{III}}=M_{\mathrm{ab}, \mathrm{a}^{\prime} \mathrm{b}^{\prime}} \pm M_{\mathrm{ab}, \mathrm{b}^{\prime} \mathrm{d}} \pm M_{\mathrm{ac}, \mathrm{a}^{\prime} \mathrm{b}^{\prime}} \pm M_{\mathrm{ac}, \mathrm{b}^{\prime} \mathrm{d}}
$$

If we now imagine the portion $a^{\prime} b^{\prime}$ to approach $a b$ and finally coincide with it, we see that $M_{\mathrm{ab}, \mathrm{b}^{\prime} \mathrm{d}^{\prime}}$ approaches $M_{\mathrm{ab}, \mathrm{bd}}$ and similarly for the other terms. Also $M_{\mathrm{ab}, \mathrm{a}^{\prime} \mathrm{b}^{\prime}}$ approaches $L_{\mathrm{ab}}$ since they are each defined as the ratio of the same voltage to the same current (divided, of course, by $\omega$ ). The mutual inductance of the two circuits thus becomes identical with that given by equation (I).

Looking at the inductance of a shunt from this point of view we note the close analogy between four-terminal resistances and mutual inductances, and in what follows we shall use the term "four-terminal impedance" to cover both classes of apparatus. As we have seen, the voltage from the potential terminals of a shunt is not, in general, in exact phase with the current, and we shall see later that, owing to eddy currents and capacity effects, the secondary voltage of a mutual inductance is not always in exact quadrature with the primary current. To avoid circumlocution, a mutual inductance which does give a secondary.voltage in quadrature with the primary current, or $\dot{a}$ shunt which has zero inductance, will be spoken of as "pure," and any departure from these ideal conditions will be described as an "impurity" in the four-terminal impedance considered. 
These definitions may be summarized in mathematical form by considering a current

$$
i=I_{\mathrm{m}} \sin \omega t
$$

to flow through the current terminals of a "four-terminal impedance." The voltage between the potential terminals may then be expressed by the equation

$$
e=E_{1} \sin \omega t+E_{2} \cos \omega t
$$

In the case of a shunt, we have the following definitions

$$
\begin{array}{ll}
\text { resistance } & R=\frac{E_{1}}{I_{\mathrm{m}}} \\
\text { inductance } & L=\frac{E_{2}}{\omega I_{\mathrm{m}}} \\
\text { phase angle } & \theta=\tan ^{-1} \frac{E_{2}}{E_{1}}=\tan ^{-1} \frac{\omega L}{R} \\
\text { time constant } & T=\frac{L}{R}=\frac{\tan \theta}{\omega}
\end{array}
$$

In the case of a mutual inductance, the corresponding definitions are

$$
\begin{aligned}
& \text { mutual inductance } M=\frac{E_{2}}{\omega I_{\mathrm{m}}} \\
& \text { "resistance" } \quad R=\frac{E_{1}}{I_{\mathrm{m}}}
\end{aligned}
$$

and, since it is more convenient to handle small angles than angles near $90^{\circ}$, we will take as the

$$
\begin{array}{ll}
\text { phase defect } & \tau=\tan ^{-1} \frac{E_{1}}{E_{2}}=\tan ^{-1} \frac{R}{\omega M} \\
\text { time constant } & T=\frac{\tan \tau}{\omega}=\frac{R}{\omega^{2} M}
\end{array}
$$




\section{RELATIVE METHODS}

\section{CURRENT-TRANSFORMER METHOD}

Measurements on the inductances of shunts may be divided into two classes: First, relative measurements in which the difference in time constant of two shunts is determined in terms of known quantities; and, second, absolute measurements in which the time constant of a single shunt is completely determined.

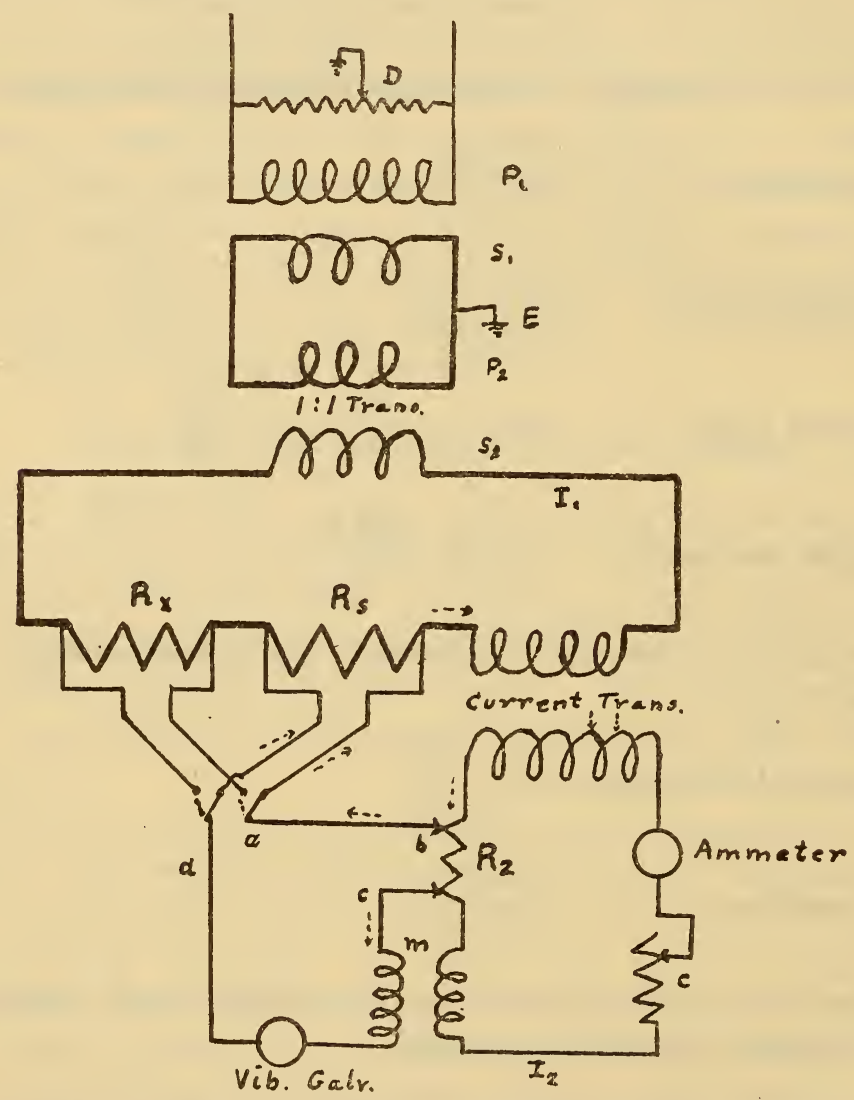

FIG. 3.-Connections for current transformer method for comparing the time constants of two shunts

A considerable variety of relative methods are available for the comparison of time constants. In addition to those mentioned on page 376 the most straightforward is perhaps the Thomson bridge as suggested by Dr. Wenner. ${ }^{11}$ This, however, is very cumbersome in practice. The method which was used in most of this work and found very rapid and convenient may be called the

${ }^{11}$ Wenner, this Bulletin, 8, p. 559, I9I2; also Sharp and Crawford, A. I. E. E. Trans., 29, p. I537, I910. 
"current-transformer method." It is a substitution method and is a highly specialized form of a-c potentiometer. The connections are shown in Fig. 3. A suitable large current $I_{1}$ flows in series through the two shunts to be compared $R_{\mathrm{s}}$ and $R_{\mathrm{x}}$ and through the primary of a current transformer. The secondary of this transformer furnishes a current $I_{2}$ which supplies the "potentiometer circuit." The voltage drop in one of the shunts is balanced against the drop in a variable resistance $R_{2}$, and any necessary quadrature component is supplied by the small variable mutual inductance $m$. The condition of balance is indicated by a sensitive vibration galvanometer. It is evident from the vector diagram (Fig. (4) that

and that

$$
\mu_{\mathrm{s}}+\theta_{\mathrm{s}}=\varphi+\theta_{2}
$$

$$
\mu=\tan ^{-1} \frac{\omega m}{R_{2}}
$$

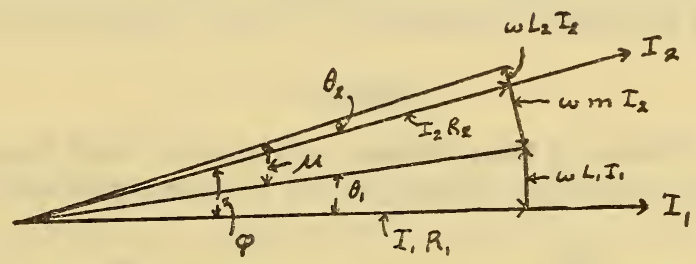

FIG. 4.-Vector diagram of current transformer method

where $\mu$ is the apparent phase angle of the transformer, $\varphi$ the true phase angle of the transformer, and $\theta_{\mathrm{s}}$ and $\theta_{2}$ the phase angles of the resistances $R_{\mathrm{s}}$ and $R_{2}$, respectively. The subscript $s$ is used here and in what follows to indicate the values observed when the standard shunt $R_{\mathrm{s}}$ is connected, while the subscript $x$ is applied to values observed when the unknown shunt $R_{\mathbf{\Sigma}}$ is used.

If the balance is repeated, using $R_{\mathbf{x}}$ we have

hence eliminating $\varphi$

$$
\mu_{\mathrm{x}}+\theta_{\mathrm{x}}=\varphi+\theta_{2}^{\prime}
$$

$$
\theta_{\mathrm{x}}-\theta_{\mathrm{s}}=\mu_{\mathrm{s}}-\mu_{\mathrm{x}}+\theta_{2}^{\prime}-\theta_{2}
$$

or

$$
T_{\mathrm{x}}-T_{\mathrm{s}}=\frac{m_{\mathrm{s}}}{R_{2}}-\frac{m_{\mathrm{x}}}{R_{2}{ }^{\prime}}+T_{2}^{\prime}-T_{2} \text { approx. }
$$

It may be noted that if the time constants of $R_{\mathrm{s}}$ and $R_{2}$ are known, this becomes a convenient method of calibrating current transformers. ${ }^{12}$

${ }^{12}$ Shatp and Crawford, A. I. E. E., 29, p. I537, I910; Agnew and Silsbee, A. I. E. E., 31, p. I267, I9I2. 
When the shunts $R_{\mathrm{s}}$ and $R_{\mathrm{x}}$ are not of equal resistance the second balance may be obtained in either of two ways. First, by simply increasing $R_{2}$, and, second, by changing the primary connections of the transformer from parallel to series. By making both these changes, shunts of very different resistance can be directly compared. The assumption made in the second method, that the phase angle of a well-designed transformer is a function of the secondary circuit conditions only, has been repeatedly justified by measurements at the Bureau of Standards. As a further check, shunts $E$ and $H$ having 0.005 and $0.0025 \mathrm{ohm}$, respectively, were compared by the two methods. The results given in Table $\mathrm{I}$ show an excellent agreement. Similar measurements at 700 cycles also agreed, showing that up to that frequency the electrostatic capacity between the windings does not affect the accuracy of the method. It is to be noted that by changing connections both shunts are kept at approximately their full current capacity and thus there is no loss of sensitivity if these capacities are different.

\section{TABLE 1}

Agreement in Difference of Time Constant of Shunts $\mathrm{H}=0.0025 \mathrm{Ohm}$ and $\mathrm{E}=0.005$ $\mathrm{Ohm} \mathrm{Obtained}$ by (a) and (b) Changing $\mathrm{R}_{2}$ and (c) Changing Ratio of Transformer.

\begin{tabular}{|c|c|c|}
\hline Ratio & $\mathbf{R}_{2}$ & $\left(T_{H}-T_{E}\right)$ obs. \\
\hline (a) $24: 1 \ldots$. & $\begin{array}{c}\text { Ohms } \\
0.12-0.060\end{array}$ & $\begin{array}{l}\text { Seconds } \\
9.89 \times 10^{-6}\end{array}$ \\
\hline (b) $12: 1 \ldots \ldots$ & $.060-.030$ & 9.92 \\
\hline (c) $24: 1-12: 1 \ldots \ldots \ldots \ldots \ldots \ldots$ & .060 & 9.93 \\
\hline Mean.. & & 9.91 \\
\hline
\end{tabular}

A special transformer was built for these measurements with ro primary coils. Each coil consisted of four parallel circuits spaced $90^{\circ}$ apart around the core. The ratio and phase angle observed when any one coil was used as primary agreed with the values observed when all ro coils were used in parallel, to within I part in 12000 in ratio and within 0.3 minute in phase angle, even when operating at a secondary current of only I ampere. The variable resistance $R_{2}$ was of Io-ampere capacity and could be adjusted to $0.0000 \mathrm{I} \mathrm{ohm.}{ }^{13}$ The mutual inductance was of the astatic type, which greatly reduced troubles from stray fields. The range needed for most work by this method is $0-5$ microhenrys.

${ }^{13}$ For description of a similar resistance, see Agnew, this Bulletin, 7, p. 423 ; I9II (Reprint No. I64). 
Since the phase angle of the transformer does depend upon the frequency, current, and secondary resistance, care must be taken to keep these constant during the comparison. A compensating resistance $C$ can be used to keep the total secondary resistance constant when $R_{2}$ is varied.

It is seen by equation (I5) that the time constants of $R_{2}$ enter into the results. This is not serious, however, since $R_{2}$ is of comparatively high resistance and small current capacity. The time constant of the apparatus used was of the order of O.I microsecond, so that the change in this from one setting to another was very slight. The value of this inductance was computed from the dimensions and the corrections applied when necessary.

The time constant $T_{2}$ of this secondary resistance may be compared with that $T_{1}$ of the primary by using a transformer having a $I: I$ ratio. Then, if the apparent phase angles $\mu_{\mathrm{a}}$ and $\mu_{\mathrm{b}}$ are observed, using first the usual connection and then one in which the primary and secondary resistances are interchanged, we get

$$
T_{1}-T_{2}=\frac{\mu_{\mathrm{b}}-\mu_{\mathrm{a}}}{2 \omega}
$$

The secondary resistances giving 0.05 and 0.10 ohm were compared with primary shunts $M$ of 0.05 ohm and $A_{2}$ of 0. Io ohm, respectively, by this method. These measurements gave $T_{\mathrm{M}}-T_{.05}=0.82 \cdot \mathrm{IO}^{-6}$ and $T_{\mathrm{A}_{2}}-T_{0.10}=2.26 \cdot \mathrm{IO}^{-8}$ second. These results are included in the general adjustment of finally accepted values given below.

The sensitivity of the method is simply a matter of the current capacity of the shunt under test and the sensitivity of the galvanometer available. Since $R_{2}$ is small and the secondary winding of $m$ can be made of low resistance, it is advisable to use a low resistance galvanometer. It is evident that under these circumstances the sensitivity is as good as it can ever be in any future normal use of the shunt with the same galvanometer. The galvanometer used in most of the work had an effective resistance of only 2 ohms and a sensitivity of $0.5 \mathrm{~mm}$ per microvolt at $60 \sim$.

The most troublesome source of error is, of course, stray fields. These, however, can be made small by keeping the heavy current circuits at a distance and then eliminated by reversing connections, etc. Another rather unexpected trouble is charging currents: These arise from two sources.' First, the relatively high $67154^{\circ}-$ vol $13-16-3$ 
voltage, say Ioo volts, used to supply the power to the primary of the step-down transformer can send sufficient charging current through the capacity of the various transformer windings to cause serious trouble. This can be avoided by ( $a$ ) grounding the source through a slide wire $D$ (see Fig. 3 ) and adjusting the potentials until the effect stops, or (b) by using a low voltage I : I transformer and grounding the primary as at $E$. A sensitive test for this effect is to open the galvanometer circuit on one side as at $a$ and then ground the secondary circuit, say at $c$. Any charging current will then flow through the galvanometer and produce a deflection. The other source of charging current is the primary of the current transformer. Currents circulate as shown by the dotted arrows, Fig. 3, and in part flow through the galvanometer. This trouble occurs only when small currents and consequently high impedances are used. It may be made negligible by keeping the side $a b$ of the galvanometer circuit of lower resistance than $c d$. Since the time constants to be measured are small and usually needed to only a few per cent, there is no difficulty in calibrating $m$ to a sufficient accuracy.

From changes in temperature, etc., the phase angle of the transformer may drift slightly during the measurements. By taking a symmetrical series of runs, first on the standard and then the unknown and vice versa, this drift can be very effectually eliminated.

In the course of this work a group of I2 shunts, some of which had several pairs of potential leads, forming the equivalent of I9 shunts, were repeatedly intercompared by this current-transformer method, making altogether some 60 difference measurements. The ig time constants were adjusted by a series of weighted averages to best fit the 60 differences. A least-square method, while theoretically more exact, would have been extremely laborious. The various individual differences were found to agree with the differences computed from the adjusted values with an average deviation of $0.05 \cdot 10^{-6}$ second. This adjustment gave, of course, only relative values of the time constants, one shunt being arbitrarily set equal to zero. A list of the shunts with their dimensions, etc., is given in Table 2. 


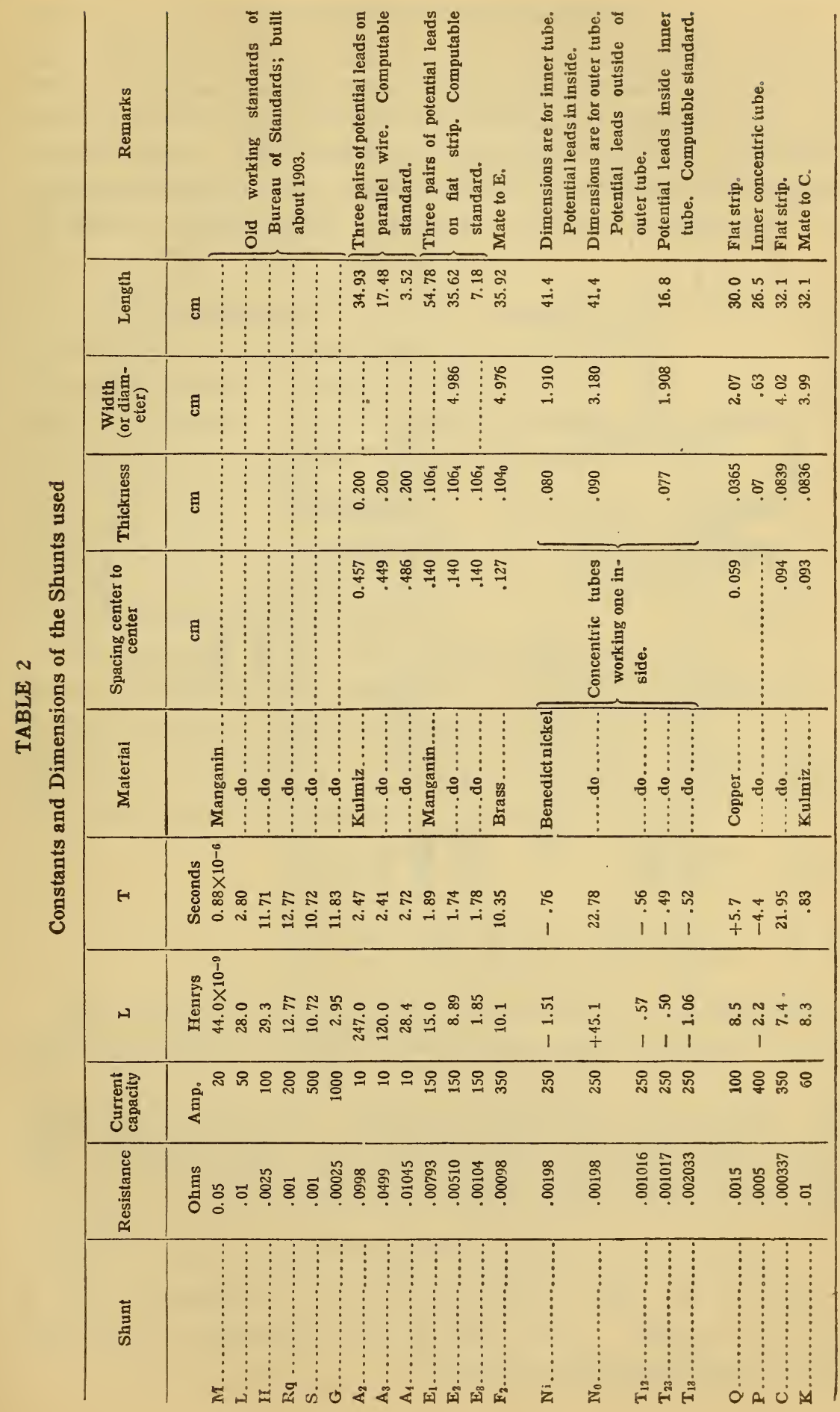


Though commercial current transformers are built only with a ratio of primary to secondary currents greater than unity, the method may be extended to shunts of less than 5 -ampere capacity by using a power transformer of suitable rating. The system of differences described above was connected with a I-ohm standard, which had been measured by Dr. Curtis of the Bureau of Standards by two-terminal methods, by such an arrangement. Using a 2

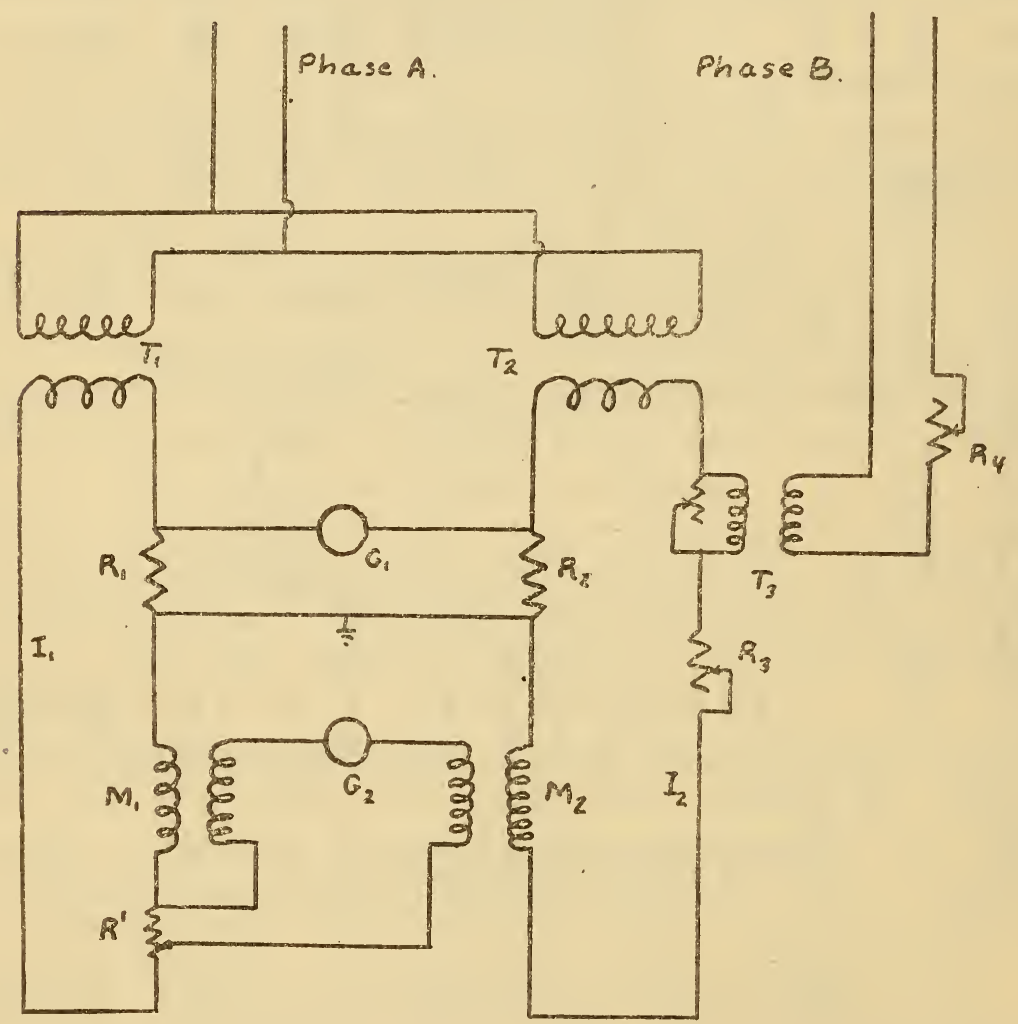

FIG. 5.-Connections for mutual inductance method for comparing the phase angles of two shunts

kW I IOO:2200/I IO-volt transformer as a step-up current transformer this I-ohm was compared with the o.I-ohm shunt $A_{2}$. The difference of their time constants was found to be

$$
T^{\mathrm{A}}{ }_{2}-T_{1 \Omega}=2.24 \times \mathrm{IO}^{-6} .
$$

This is in good agreement with the computed value of 2.18. $\mathrm{IO}^{-6}$ seconds. The results of this comparison are included in the final adjustment in Table 9. 


\section{MUTUAL-INDUCTANCE METHOD}

A second relative method was tried in this work. It is also of the potentiometer type, but involves a simultaneous balance on the standard and unknown shunts instead of a substitution of one for the other. The connections are shown in Fig. 5. Here $R_{1}$ and $R_{2}$ are the shunts under test and $M_{1}$ and $M_{2}$ two mutual inductances. Phase A of a twophase generator supplies through two transformers $T_{1}$ and $T_{2}$ the currents $I_{1}$ and $I_{2}$ which are approximately in the same phase. The other phase, $B$, excites the small transformer $T_{3}$ and thus inserts into the secondary of $T_{2}$ a variable out-of-phase component and permits of a sensitive control of the relative phases of $I_{1}$ and $I_{2}$. The magnitude of $I_{2}$ is regulated by the resistance $R_{3}$.

The procedure is for one observer to control $R_{4}$ and $R_{3}$ until $G_{1}$ shows a balance while the other

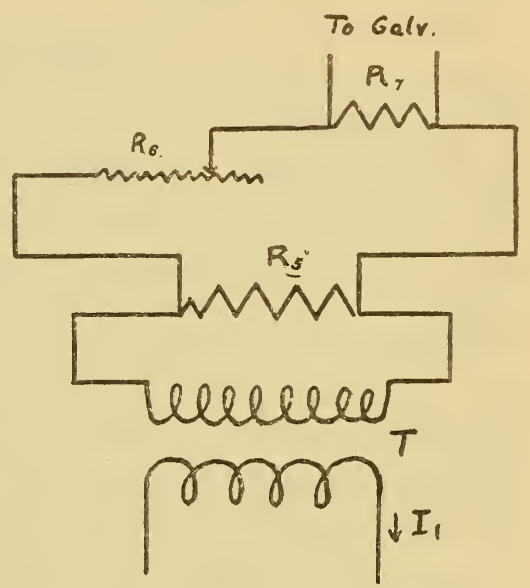

FIG. 6.-Connections for obtaining a very small adjustable resistance observer varies $R^{\prime}$ and $M_{1}$ until $G_{2}$ is balanced. When such a simultaneous balance has been obtained we have, neglecting terms of higher order, the relations:

$$
\begin{aligned}
& \frac{M_{1}}{M_{2}}=\frac{R_{1}}{R_{2}} \\
& \frac{L_{2}}{R_{2}}-\frac{L_{1}}{R_{1}}=\frac{R^{\prime}}{\omega^{2} M_{1}}+\frac{I}{\omega}\left(\tau_{1}-\tau_{2}\right)^{14}
\end{aligned}
$$

The voltages in the circuit of galvanometer $G_{1}$ are determined by the permissible voltage on the shunts, and $G_{1}$ should have as low resistance as possible. The magnitudes of $M_{1}$ and $M_{2}$, however, are arbitrary, subject to equation ( 17 ). Hence, this circuit can be designed to give higher sensitivity than the other and therefore will introduce no observational errors in the result. The resistance $R^{\prime}$ must be very small. The equivalent of a very small resistance can most easily be obtained by the arrangement shown in Fig. 6. If $R_{5}$ and $R_{7}$ are small compared with $R_{6}$ we have

$$
R^{\prime}=\frac{R_{7} R_{5}}{R_{6}} \cdot \frac{I}{P}
$$


where $P$ is the ratio of the current transformer $T$. The transformer is not essential, but helps reduce $R^{\prime}$ and also insulates the galvanometer circuit and greatly reduces stray charging currents. The effect of the phase angle of the transformer is entirely negligible. In making a setting care should be taken to vary $R_{6}$ and not $R_{7}$. Otherwise, the change in sensitivity may lead to an entirely false minimum.

Stray fields and charging currents must, of course, be guarded against as in all work of this kind. A more serious source of error is the correction $\left(\tau_{1}-\tau_{2}\right)$ due to the phase defects of the mutual inductances used. If the two shunts are of equal resistance, the inductances may be interchanged and the correction thus determined. The determination of this correction in the general case will be discussed more fully later.

Four difference measurements were made by this method and the results are given in Table 3 , together with the values computed from observations by the current-transformer method. It is seen that this mutual-inductance method is not as precise as the former, but that there is no constant discrepancy greater than the experimental error of the latter. The lack of precision is probably due to the continual variations in resistance of the two heavy current circuits caused by self-heating, drafts, etc. This fluctuation requires the observers to be constantly alert and makes the simultaneous balance rather difficult.

TABLE 3

Results of Relative Measurements by Mutual-Inductance Method

\begin{tabular}{|c|c|c|c|}
\hline Shunts compared & $\begin{array}{c}\left(\mathrm{T}_{1}-\mathrm{T}_{2}\right) \\
\text { Mutual inductance } \\
\text { method }\end{array}$ & $\begin{array}{l}\left(T_{1}-T_{s}\right) \\
\text { Current trans- } \\
\text { former method }\end{array}$ & Difference \\
\hline & Microseconds & Microseconds & Microseconds \\
\hline $\mathbf{R}-\mathbf{S} \ldots$. & 2.1 & $2.0_{5}$ & +0.0 \\
\hline $\mathbf{G}-\mathbf{T}_{13} \ldots \ldots \ldots$ & 12.1 & $12.3_{3}$ & $-.2 \overline{3}$ \\
\hline G-L................................ & 9.0 & $9.0_{3}$ & $-.0_{3}$ \\
\hline $\mathbf{L}-\mathrm{T}_{13} \ldots \ldots \ldots \ldots \ldots \ldots \ldots \ldots \ldots$ & 3.8 & $3.3_{2}$ & +.48 \\
\hline
\end{tabular}

\section{ABSOLUTE METHODS}

The best method for the determination of the inductance of $a$ shunt is by comparison with some four-terminal standard whose inductance can be computed from the dimensions. This method, which is the only one hitherto used, is open to the objection that in the computed standard the current distribution, end 
effects, etc., may not be such as are assumed in the mathematical theory. In the work described below each type of standard was carefully studied with a view to these sources of error. Also three distinct types were used and the probability of the same error occurring in all is very remote. Nevertheless, it was thought advisable to use two other methods recently proposed, ${ }^{15}$ in which there is no dependence on computation.

\section{COMPUTATION}

There are many forms of circuit, the inductance of which can be computed from the shape and dimensions of the conductors. For the purpose of this investigation, however; where it was necessary to keep the ratio $\frac{L}{R}$ small, the available forms are all of the type of two parallel straight conductors, long in comparison with their cross section and spacing.

Formulas for the inductance of such circuits have been given by Gray, ${ }^{16}$ Rosa, ${ }^{17}$ Orlich, ${ }^{18}$ and others. For shunts of type $I$ in which the resistance material forms a nearly closed circuit, these formulas are special cases of the general expression

$$
L=2 l\left(2 \log D_{12}-\log D_{11}-\log D_{22}\right)
$$

where $D_{12}$ is the geometric mean distance of the cross-sectional areas of the conductors from each other and $D_{11}$ and $D_{22}$ the g. m. d's of each area from itself. For shunts of type II it is necessary to either build up the inductance piecemeal as indicated by equation (I) or to regard it as the limit of the mutual inductance of two circuits which coincide over a part of their length.

These formulas are deduced on the assumption that the current is uniformly distributed over the cross section of the conductor. That this limitation is not as serious as appears at first sight is shown by the followng reasoning: The emf induced by the magnetic field is not in general the same at different points in the cross section of the conductor and therefore the current density will not be uniform over the section. The resulting current distribution may be looked upon as made up of a system of eddy currents superposed on a uniform distribution. The net effect of the resistance drop due to these currents and the induced emf must be to make the planes perpendicular to the axis equipotential.

15 Wenner, Weibel, and Silsbee, this Bulletin, 12, p. II; I9I5 (Reprint No. 246).

${ }^{16}$ Gray, Absolute Measurements, Vol. II, Part I, p. 288.

17 Rosa, this Bulletin 4, p. 321, 1907; Rosa and Grover, this Bulletin 8, p. I50, I9II.

18 Orlich, Kapazität und Induktivität, p. 68. 
If this is the case the quadrature component of the resultant potential difference per unit length is

$$
e_{\mathrm{o}}=e-i_{\mathrm{e}} \rho
$$

where $e$ is the induced linear emf, $i_{\mathrm{e}}$ the eddy-current density, and $\rho$ the resistivity. Multiplying by $d S$ and integrating over the whole cross section of the conductor

$$
\int e_{\mathrm{o}} d S=\int e d S-\int \rho i_{\mathrm{e}} d S
$$

but since $i_{\mathrm{e}}$ is merely the eddy-current density $\int i_{\mathrm{e}} d S$ must vanish

$$
\therefore e_{0}=\frac{\int e d S}{S}
$$

This area average of induced emf is what is given by the geometric mean distance formulas and they are therefore valid even if the increase in resistance due to skin effect is appreciable provided only that the magnetic effects of the eddy currents are negligible.

It is sometimes more convenient to go back to this area average of emf in computing inductances than to use geometric mean distances, and this method will be used in developing the formulas for tubular shunts given below.

(a) Parallel Wire Type--For the case of parallel wires equation (I9) becomes

$$
\begin{aligned}
L & =4 l\left(\log \frac{d}{a}+\frac{\mathrm{I}}{4}\right) \\
d & =\text { spacing of wires center to center } \\
a & =\text { radius of wire. }
\end{aligned}
$$

This formula assumes uniform current distribution over the cross section, but there is little liability to error from this source at commercial frequencies. Any nonhomogeneity due to the process of drawing would leave the resistivity symmetrical about the axis and affect only the small second term.

Shunt "A" was constructed of Kulmiz alloy wires $0.200 \mathrm{~cm}$ in diameter and spaced $0.457 \mathrm{~cm}$ center to center. Pairs of potential leads were attached at distances of $3.52, I 7.5$, and $35 \mathrm{~cm}$ from the closed end. Difference measurements were made by the current transformer method and showed that the end effects were entirely negligible. Table 4 gives in the second column the values of $T$ computed from the dimensions and the observed resistance assuming an undetermined inductance $\Delta L$ to represent the end effect. Comparing the differences of these values with the observed differences, we see that setting $\Delta L$ equal to zero gives the best agreement. 
TABLE 4

Results on Shunt A

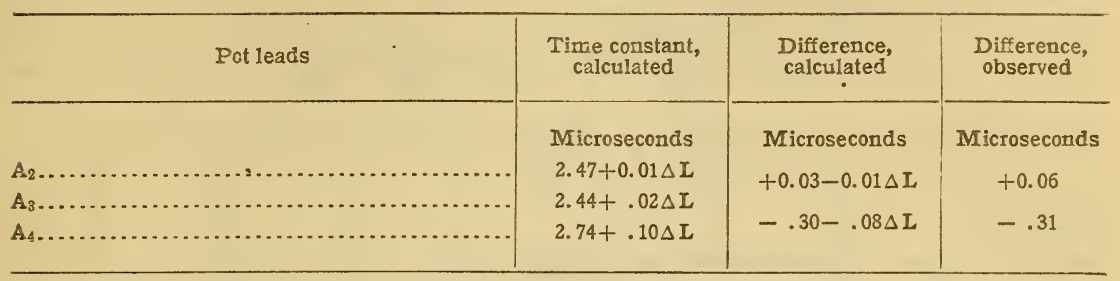

(b) Flat Strip Type.-In the case of parallel rectangular conductors the exact formula ${ }^{19}$ obtained from equation (I9) is inconvenient, not only because of the large number of terms, but also because the result appears as the difference of large terms, which must, thefefore, be computed with great accuracy. By expanding in series, however, it can be put in the much more convenient form ${ }^{20}$

$$
\begin{aligned}
L & =4 l\left[\frac{\pi}{3}(3 \beta-\delta)-\frac{25}{\mathrm{I} 2} \beta^{2}-\frac{\mathrm{I}}{\mathrm{I} 2} \beta^{4}-\frac{\mathrm{I}}{\mathrm{I} 2} \beta^{2} \delta^{2} \ldots \ldots\right. \\
& \left.+\frac{\mathrm{I}}{\mathrm{I} 2 \delta^{2}}\left\{\alpha^{4} \log \alpha-2 \beta^{4} \log \beta+\gamma^{4} \log \gamma-2 \delta^{4} \log \delta\right\}\right]
\end{aligned}
$$

Here

where

$$
\alpha=\frac{2 b+g}{w}, \beta=\frac{b+g}{w}, \gamma=\frac{g}{w}, \delta=\frac{b}{w}
$$

$$
\begin{aligned}
w & =\text { width of strip } \\
b & =\text { thickness of metal } \\
g & =\text { thickness of insulator } \\
l & =\text { length of circuit }
\end{aligned}
$$

The terms neglected in this formula are of the order $\beta^{8}$.

In case the strips are of unequal thickness we have the analogous expression ${ }^{21}$

$$
\begin{array}{r}
L_{1}=2 l\left[\pi\left(\gamma+\frac{\eta}{2}+\frac{\delta}{6}\right)-25\left(\frac{\gamma \alpha}{\mathrm{I} 2}+\frac{\eta^{2}}{36}+\frac{\delta \eta}{24}+\frac{\delta^{2}}{72}\right) \ldots .\right. \\
\left.+\frac{\mathrm{I}}{\mathrm{I} 2 \delta \eta}\left(\alpha^{4} \log \alpha-\kappa^{4} \log \kappa-\lambda^{4} \log \lambda+\gamma^{4} \log \gamma-2 \delta^{3} \eta \log \delta\right)\right]
\end{array}
$$

19 The value of $D_{12}$ is given by Rosa, this Bulletin 3, p. 6 eq. (8), and for $D_{11}$, this Bulletin 8 , p. I67 eq. (I24).

${ }^{20}$ An equivalent form is given by Orlich (Zs. für Instrumentenkunde 29, p. 24I; 1909), together with a table of values of $x^{4} \log x$, which is useful in computing the last term. The formula may be obtained by substituting into eq. ( $\mathrm{rg}$ ) the proper values of $D_{12}$ and $D_{11}$. The series expansion is obtained by expressing the logarithmic and antitrigonometric terms as power series in $\alpha, \beta$, etc. The terms may then be combined and the higher powers neglected.

21 The derivation of this equation is similar to that of eq. 24 except that the values for the geometric mean distance are substituted in $L_{1}={ }_{2} l \log \frac{D_{12}}{D_{11}}$, since the inductance of a single strip is desired instead of that of the return circuit. 
Here $L_{1}$ is the inductance of the strip having thickness $b$ with return in the other strip, where

where

$$
\begin{aligned}
& \alpha=\frac{b+c+g}{w}, \gamma=\frac{g}{w}, \delta=\frac{b}{w}, \eta=\frac{c}{w} \\
& \kappa=\frac{b+g}{w}, \lambda=\frac{c+g}{w}
\end{aligned}
$$

$$
\begin{aligned}
& w=\text { width of strips } \\
& b=\text { thickness of strip used } \\
& c=\text { thickness of return conductor } \\
& g=\text { thickness of insulator } \\
& l=\text { length of circuit. }
\end{aligned}
$$

An interchanging of the letters $\delta$ and $\eta$ gives, of course, the inductance $L_{2}$ of the other strip. The inductance of the complete circuit is then the sum $L_{1}+L_{2}$.

These formulas were used in computing the corrections for the inductance of the secondary resistance $R_{2}$ mentioned on page 385 above.

A standard shunt $E$ was constructed of sheet manganin 0.I06 $\mathrm{cm}$ thick, $4.99 \mathrm{~cm}$ wide, and about $60 \mathrm{~cm}$ long. The strips were clamped firmly together with $0.035 \mathrm{~cm}$ of paper insulation between. Pairs of potential leads were attached at distances $7.2,35.6$, and $54.8 \mathrm{cms}$. from the closed end, giving resistances of $0.001,0.005$, and $0.008 \mathrm{ohm}$, respectively.

The sources of error to be guarded against in a shunt of this type are (I) skin effect, (2) nonuniform current distribution due to lack of homogeneity, (3) inductive effects in the potential leads, (4) end effects. These points will be considered in order. Bethenod, ${ }^{22}$ Orlich, ${ }^{23}$ and others have computed the effect of nonuniform current distribution on the resistance and inductance of a circuit of this shape assuming the length and width of the plates both infinite. The latter assumption seems a little startling, but since the strips are close together the magnetic field is nearly constant over most of the width. The nonuniform distribution edgeways is confined to a slight excess of current over a region at the edge only a few per cent of the width of the plate. These formulas show that for the frequencies and materials used the skin effect is entirely negligible.

A more serious possibility is the danger of nonuniform current distribution due to accidental causes, such as poor solder, where the

${ }^{22}$ Resistance of Thin Plates to alternating currents, Jahr. d. Drahtloser Tel., 2, p. 397.

${ }^{22}$ Zeitschrift für Instrumentenkunde, 29, p. 24I; I909. 
current leads are attached and nonhomogeneity in the material itself. If, for example, the current tended to flow in the upper edge of one sheet and the lower edge of the other, this would seriously alter the inductance. The theoretical formula for the

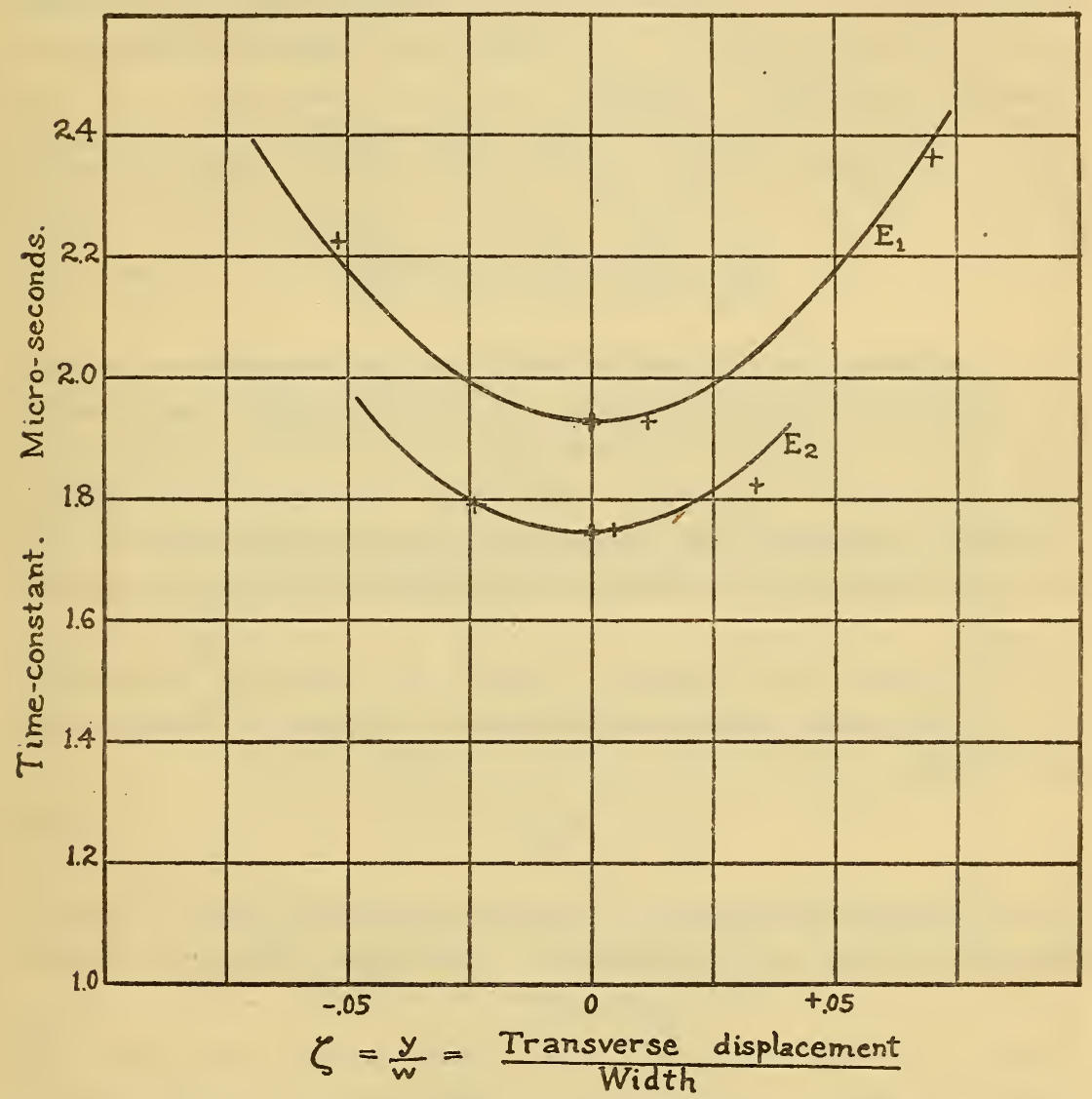

FIG. 7.-Effect of transverse displacement on inductance of flat strip shunt. Curves are computed, crosses are observed points

change in linear inductance of two infinitely thin strips of width $w$ and spacing $d$ when displaced transversely a distance $y$ is

$\Delta L=4\left[\left[\frac{3}{2} \xi^{2}-\frac{1}{2}\left(\zeta^{2}-\beta^{2}\right) \log \left(\zeta^{2}+\beta^{2}\right)-\beta^{2} \log \beta-2 \beta \zeta \tan ^{-1} \frac{\zeta}{\beta}\right]\right.$

where $\zeta=\frac{y}{w}$ and $\beta=\frac{d}{w}$. This effect always tends to increase the inductance, and the procedure adopted was to slide the strips past each other (by slightly springing the closed end) and measure the inductance in each position. The curves on Fig: 7 are computed 
from eq (26) and the crosses (+) are the observed points. They seem to indicate that the true zero, where the currents most nearly neutralize, is slightly to the right of the mechanical zero. The difference is very slight, however, and was neglected. "Though this agreement of the theoretical and observed values is of course no proof that the current is uniformly distributed, it indicates that such is probably the case.

The end effects and mutual inductance errors were handled in the same manner as with shunt $A$. The difference measurements gave

$$
\begin{aligned}
& T_{\mathrm{E}_{1}}-T_{\mathrm{E}_{2}}=0.17 \cdot 10^{-6} \mathrm{sec} . \\
& T_{\mathrm{E}_{3}}-T_{\mathrm{E}_{2}}=0.04 \cdot 10^{-6} \mathrm{sec} .
\end{aligned}
$$

The first difference is probably due to mutual inductance between the current and potential leads. Since the leads $E_{2}$ were seven times as far from the current leads as $E_{1}$, any such effect in $E_{2}$ would be entirely negligible. The second difference is practically negligible and brings out the fact that the formulas given above hold for shunts nearly as short as their width, provided the spacing is close.

To determine the precision required in measuring the dimensions of the shunt, equation (24) may be written in the approximate form

$$
L=4 \pi \frac{l}{w}\left(a-\frac{4}{3} b\right)
$$

where the over-all dimension $a$ and the thickness of metal $b$ are the dimensions most easily measured. By differentiating we obtain

$$
\frac{d L}{L}=\frac{d a}{a} \cdot \frac{\mathrm{I}}{\mathrm{I}-\frac{4}{3} \cdot \frac{b}{a}}
$$

and

$$
\frac{d L}{L}=-\frac{d b}{b} \cdot \frac{\mathrm{I}}{\frac{3}{4} \cdot \frac{a}{b}-\mathrm{I}}
$$

In the limiting cases of $b=0$ and $b=\frac{a}{2}$

these become

$$
\frac{d L}{L}=\frac{d a}{a} \cdot \mathrm{I} \text { and } \frac{d L}{L}=\frac{d a}{a} \cdot 3
$$

and

$$
\frac{d L}{L}=-\frac{d b}{a} \cdot \frac{4}{3} \text { and } \frac{d L}{L}=-\frac{d b}{b} \cdot 2
$$


respectively. The percentage error in $L$ is therefore at worst three times the percentage error in the measurement of $a$ and twice the percentage error in $b$. Since these may be conveniently measured to a fraction of I per cent with micrometer calipers, they are not particularly serious sources of error.

(c) Tubular Type.-The third type of shunt which can be used as a computable standard consists of a pair of concentric tubes one or both of which are of resistance material. The potential leads may be arranged in a number of different ways yielding correspondingly different values of the four-terminal inductance. The formulae for the various cases are so similar in

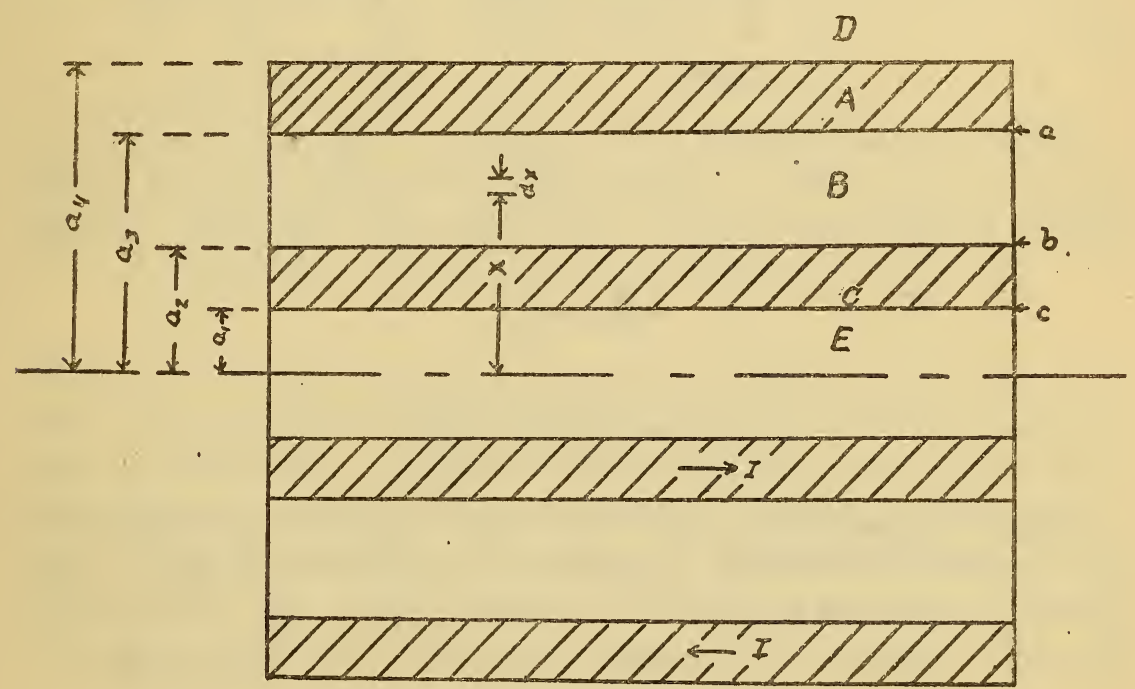

FIG. 8.-Cross section of tubular shunt

appearance and the self and mutual inductive effects so superposed that an analysis from the closed-circuit point of view is very confusing. The following discussion will separate the various sources of emf and obtain any particular case by a summation of the proper effects.

Fig. 8 represents a longitudinal section of unit length of such a pair of concentric tubes. Let the radii be $a_{1}, a_{2}, a_{3}$, and $a_{4}$ from inside outward,

and let us define

and

$$
\left.\begin{array}{c}
s=\frac{a_{4}-a_{3}}{a_{4}} \\
t=\frac{a_{2}-a_{1}}{a_{2}} \\
u=\frac{a_{3}-a_{2}}{a_{3}}
\end{array}\right\}
$$

and 
If a current $I$ flows to the right in the inner tube and returns in the outer, the magnetic flux densities $H_{\mathbf{D}}, H_{\mathbf{A}}$, etc. (assuming $\mu=\mathrm{I}$ and the tubes infinitely long) in the regions $D, A, B, C, E$ are, respectively,

$$
\begin{aligned}
& H_{D}=0 \\
& H_{\mathrm{A}}=\left\{\frac{2}{x}-\frac{2\left(x^{2}-a_{3}{ }^{2}\right)}{x\left(a_{4}{ }^{2}-a_{3}^{2}\right)}\right\} I \\
& H_{\mathrm{B}}=\frac{2}{x} I \\
& H_{\mathrm{C}}=\frac{2\left(x^{2}-a_{1}^{2}\right)}{x\left(a_{2}{ }^{2}-a_{1}^{2}\right)} I \\
& H_{\mathrm{E}}=0
\end{aligned}
$$

Where $x$ is the distance from the axis to the point considered. It will be convenient to split $H_{\mathrm{A}}$ into two parts, $H_{\mathrm{Ar}}$, due to the current in the inner, and $H_{\mathrm{AO}}$, due to the outer conductor. Where

$$
\begin{gathered}
H_{\mathrm{AI}}=\frac{2}{x} I \\
H_{\mathrm{AO}}=-\frac{2\left(x^{2}-a_{3}{ }^{2}\right)}{x\left(a_{4}{ }^{2}-a_{3}{ }^{2}\right)} I
\end{gathered}
$$

Integrating equations (33) over their respective areas gives us the complete flux linking a conductor just inside each area, which we may denote by $\phi_{\mathrm{AO}_{2}}$, etc., i. e., the flux in $A$ due to current in the outer conductor and linking a wire at $a$. We thus obtain

$$
\begin{aligned}
\phi_{\mathrm{AO}} & =\int a_{3}^{a_{4}} H_{\mathrm{AO}} d x=\left[-\mathrm{I}+\frac{2 a_{3}{ }^{2}}{a_{4}{ }^{2}-a_{3}{ }^{2}} \log \frac{a_{4}}{a_{3}}\right] I \\
& =\left(-s-\frac{s^{2}}{6}+\frac{s^{4}}{30} \cdots\right) I \\
\phi_{\mathrm{AI}} & =\int a_{a_{3}}^{a_{4}} H_{\mathrm{AI}} d x=\left[2 \log \frac{a_{4}}{a_{3}}\right] I=\left(2 s+s^{2}+\frac{2}{3} s^{3} \cdots\right) I \\
\phi_{\mathrm{BIb}} & =\int a_{3}^{a_{3}} H_{\mathrm{BI}} d x=\left[2 \log \frac{a_{3}}{a_{2}}\right] I=\left(2 u+u^{2}+\frac{2}{3} u^{3} \cdots\right) I \\
\phi_{\mathrm{CI}} & =\int a_{a_{1}}^{a_{2}} H_{\mathrm{CI}} d x=\left[I-\frac{2 a_{1}{ }^{2}}{a_{2}{ }^{2}-a_{1}{ }^{2}} \log \frac{a_{2}}{a_{1}}\right] I=\left(+t+\frac{t^{2}}{6}-\frac{t^{4}}{30} \cdots\right) I
\end{aligned}
$$

We shall also need expressions for $\phi_{\mathrm{AOA}}$, i. e., average linkages of the flux in $A$ due to the outer current linking the conductor $A$ 
and producing an average voltage as defined above. These expressions are given by

$$
\begin{aligned}
\phi_{\mathrm{AOA}} & =\frac{\mathrm{I}}{\pi\left(a_{4}{ }^{2}-a_{3}{ }^{2}\right)} \int_{a_{3}}^{a_{4}} 2 \pi x d x \int_{x}^{a_{4}} H_{\mathrm{AO}} d x \\
& =\left[-\frac{a_{4}{ }^{2}-3 a_{3}{ }^{2}}{2\left(a_{4}{ }^{2}-a_{3}{ }^{2}\right)}-\frac{2 a_{3}{ }^{4}}{\left(a_{4}{ }^{2}-a_{3}{ }^{2}\right)^{2}} \log \frac{a_{4}}{a_{3}}\right] I \\
& =\left(-\frac{2}{3} s+\frac{\mathrm{I}}{\mathrm{I} 5} s^{3}+\frac{s^{4}}{20} \cdots\right) I
\end{aligned}
$$

$$
\begin{aligned}
\oint_{\mathrm{AIA} A} & =\frac{\mathrm{I}}{\pi\left(a_{4}^{2}-a_{3}^{2}\right)} \int a_{a_{3}}^{a_{4}} 2 \pi x d x \int_{x}^{a_{4}} H_{\mathrm{AI}} d x \\
& =\left[-\frac{2 a_{3}{ }^{2}}{a_{4}{ }^{2}-a_{3}{ }^{2}} \log \frac{a_{4}}{a_{3}}+\mathrm{I}\right] I=\left(+s+\frac{s^{2}}{6}-\frac{s^{4}}{30} \cdots\right) I
\end{aligned}
$$

$$
\begin{aligned}
\phi_{\mathrm{CIC}} & =\frac{\mathrm{I}}{\pi\left(a_{2}^{2}-a_{1}^{2}\right)} \int_{a_{1}}^{a_{2}} 2 \pi x d x \int_{x}^{a_{2}} H_{\mathrm{CI}} d x \\
& =\left[\frac{a_{2}^{2}-3 a_{1}^{2}}{2\left(a_{2}^{2}-a_{1}{ }^{2}\right)}+\frac{2 a_{1}^{4}}{\left(a_{2}{ }^{2}-a_{1}{ }^{2}\right)^{2}} \log \frac{a_{2}}{a_{1}}\right] I=\left(\frac{2}{3} t-\frac{t^{3}}{\mathrm{I} 5}-\frac{t^{4}}{20} \cdots\right) I
\end{aligned}
$$

It may be noted that $\phi_{\mathrm{AO}}=-\phi_{\mathrm{AIA}}$ as should be the case since they are expressions for the mutual inductance between the same pair of conductors. In each of these equations the approximate expression in $s, t$, or $u$ may be obtained by a simple series expansion. Since in practice the tubes used are always thin in comparison with their radii, these approximate expressions are sufficiently accurate and very convenient.

Considering now a few special cases, let us first take the ordinary "concentric main," as in Fig. $9 a$, where both tubes are used as resistance material and the potential leads are in the same transverse plane. Adding the various linkages with regard to their directions we get

$$
\begin{array}{rl}
L_{\mathrm{a}}= & {\left[\phi_{\mathrm{CIC}}+\phi_{\mathrm{BIb}_{\mathrm{b}}}+\phi_{\mathrm{AI}_{\mathrm{a}}}-\phi_{\mathrm{AOA}}+2 \phi_{\mathrm{AO}}\right] \frac{l}{I}} \\
=l & l-\frac{a_{2}{ }^{2}+a_{1}{ }^{2}}{2\left(a_{2}{ }^{2}-a_{1}{ }^{2}\right)}-\frac{a_{4}{ }^{2}+a_{3}{ }^{2}}{2\left(a_{4}{ }^{2}-a_{3}{ }^{2}\right)}+\frac{2 a_{1}{ }^{4}}{\left(a_{2}{ }^{2}-a_{1}{ }^{2}\right)^{2}} \log \frac{a_{2}}{a_{1}} \\
& \left.\quad+\frac{2 a_{4}{ }^{4}}{\left(a_{4}{ }^{2}-a_{3}{ }^{2}\right)^{2}} \log \frac{a_{4}}{a_{3}}+2 \log \frac{a_{3}}{a_{2}}\right]
\end{array}
$$

or

$$
L=l\left[\frac{2}{3} t+2 u+\frac{2}{3} s+u^{2}+\frac{2}{3} s^{2} \cdots\right] \text { (approx.) }
$$


This formula is identical with that deduced by Russell and others. This form of shunt, however, is not well adapted for use as a standard, since it requires two soldered joints between the potential terminals, and also there is an end correction which must be obtained experimentally.

A better arrangement is to use either the outer or inner tube separately, thus forming a shunt of the second type. In the latter case the potential leads may be brought out either radially through
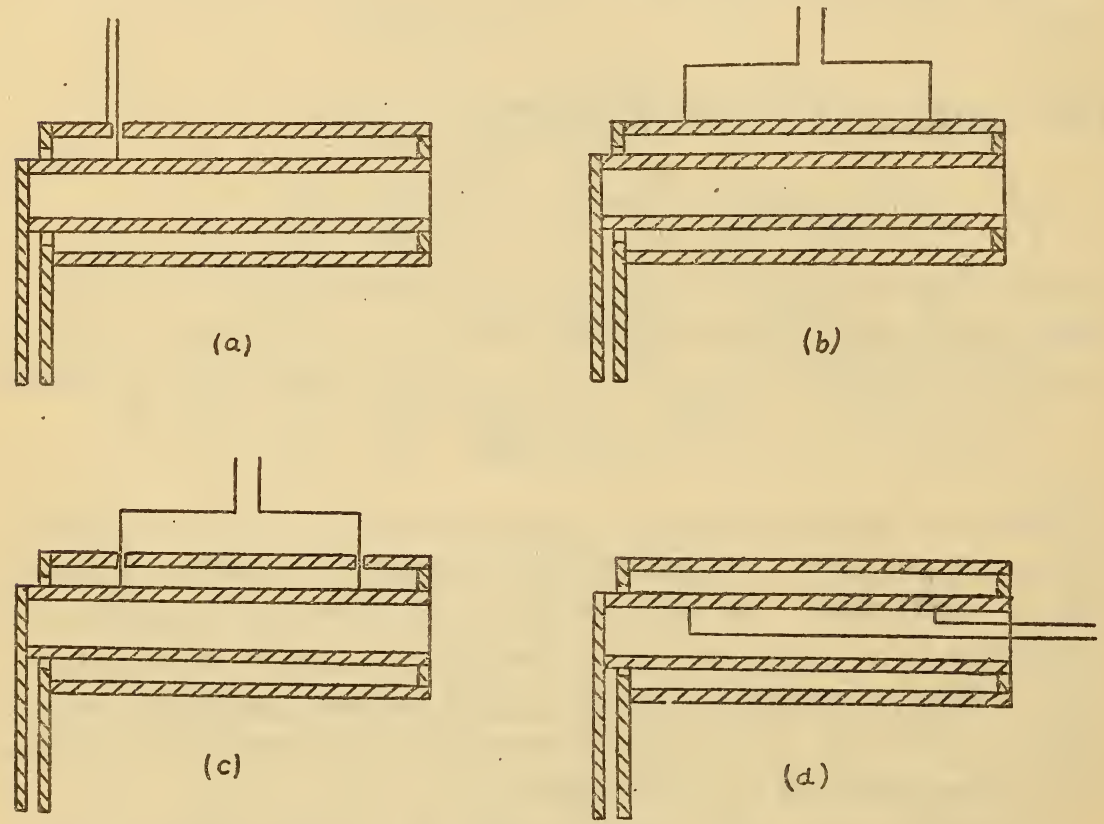

FIG. 9.-Various arrangements of poteniial leads in tubular shunts

insulated openings in the outer tube as in 9 (c) or axially as in $9(d)$. The inductances per unit length in the three cases are

$$
\begin{aligned}
L_{(\mathrm{b})} & =\left[-\phi_{\mathrm{AIA}}-\phi_{\mathrm{AOA}}\right] \frac{l}{I}=\left(-\frac{\mathrm{I}}{3} s-\frac{\mathrm{I}}{6} s^{2} \cdots\right) l \\
L_{(\mathrm{c})} & =\left[\phi_{\mathrm{CIC}}+\phi_{\mathrm{BIb}}+\phi_{\mathrm{AO}_{\mathrm{a}}}+\phi_{\mathrm{AI}}\right] \frac{l}{I} \\
& =\left(s+\frac{2}{3} t+2 u+\frac{5}{6} s^{2}+u^{2} \cdots\right) l \\
L_{(\mathrm{d})} & =\left[\phi_{\mathrm{CIC}}+\phi_{\mathrm{BIb}}+\phi_{\mathrm{AO} a}+\phi_{\mathrm{AI}_{\mathrm{a}}}-\phi_{\mathrm{CI}_{\mathrm{c}}}-\phi_{\mathrm{BIb}}-\phi_{\mathrm{AI}_{\mathrm{a}}}-\phi_{\mathrm{AO} \mathrm{a}}\right] \frac{l}{I} \\
& =\left[\phi_{\mathrm{CIC}}-\phi_{\mathrm{CI}_{\mathrm{C}}}\right] \frac{l}{I}=\left(-\frac{t}{3}-\frac{t^{2}}{6} \cdots\right) l
\end{aligned}
$$


It may be noted that both $L_{\mathrm{b}}$ and $L_{\mathrm{d}}$ are negative and smaller in magnitude than $L_{\mathrm{a}}$ or $L_{\mathrm{c}} . L_{\mathrm{c}}$ is also of interest because, although the working material does not form a closed circuit and the shunt properly belongs in the second type, yet there is no electromotive force induced in the potential leads.

A shunt $N$ was constructed with a "Benedict nickel" tube inside a brass return tube, and so arranged that they could be shifted transversely. The resistance was about $0.002 \mathrm{ohm}$. Two sets of potential leads were used. " $\mathrm{N}_{\mathrm{o}}$ " arranged as in Fig. 9 (c) and " $N_{i}$ " as in $(d)$. The inductances of the two arrangements were $45 . \mathrm{I} \times \mathrm{IO}^{-9}$ henry and $-\mathrm{I} .5 \times 1 \mathrm{IO}^{-9}$ henry, respectively.

At first sight it would appear that the inductance would be affected by any eccentricity of the tubes, but since the geometric mean distance of a point inside a circular annulus is independent of the position of the point it is evident from equation (I9) that the inductance is also independent of eccentricity. This assumes that the current in the outer tube is uniformly distributed and that the tube is circular. The latter requirement is readily met by commercial tubing, but the former is difficult to obtain.

If the distribution in both tubes is uniform arourd the circumference, there is no magnetic flux inside the inner tube, and therefore an exploring coil inserted in this tube forms a very sensitive test for such a lack of uniformity. Shunt $N$ showed a magnetic field at the center equivalent to that at a distance of $\mathrm{I} \mathrm{cm}$. from a wire carrying 3 per cent of the full current. This nonuniformity could not be eliminated by filing the ends of the outer tube and was probably due to lack of homogeneity in the brass. Since the inner potential leads were placed at the axis of the tube even this field should have had no effect on the inductance. This was corroborated by the fact that an extra current equal to ro per cent of the main current flowing in an auxiliary wire just outside the shunt produced a barely detectible effect.

The effect of eccentricity was also investigated with this shunt, but a displacement as great as $1.5 \mathrm{~mm}$ showed no change in the time constant of $N_{i}$ greater than 0.05 microsecond.

Heaviside $^{24}$ has shown that the rate of change in inductance of a concentric main with frequency is very small, involving only terms of the third order. Shunt $T$ was also constructed in this shape, but using a copper tube of very uniform cross section as the return. The lack of uniform current distribution was found to be only onethird as serious as with shunt $N$ and the inductance is believed to be very closely equal to the theoretical value. 


\section{QUARTER-PHASE METHOD}

Though the values of inductance obtained from computable standards were believed to be correct, it was considered advisable to obtain an independent check if possible. Two such direct methods of measurement had been suggested by Dr. Wenner and

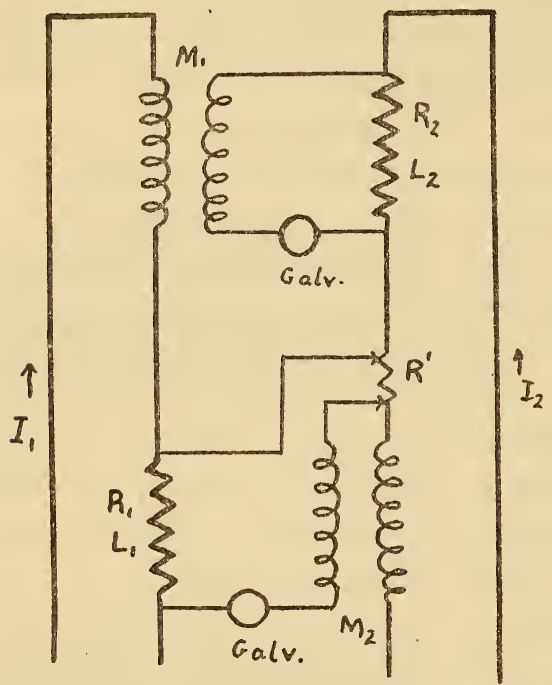

Fig. Io.-Connections for quarter-phase method for measuring the inductance of shunts

Mr. Weibel of the Bureau of Standards and were tried out in some preliminary measurements in conjunction with the author. ${ }^{25}$

The first of these methods gives the sum of the time constants of the two shunts to be compared. The connections are shown in Fig. ro. $M_{1}$ and $M_{2}$ are mutual inductances whose primary windings have the same current carrying capacity as the shunts. The two circuits $A$ and $B$ are excited by alternating currents approximately in quadrature, and the values of $M$ and $R^{\prime}$ and the relative phase and magnitude of $I_{1}$ and $I_{2}$ are adjusted until a simultaneous balance on both galvanometers is obtained. We then have neglecting small terms the two relations

$$
\begin{aligned}
& R_{1} R_{2}=\omega^{2} M_{1} M_{2} \\
& \frac{L_{1}}{R_{1}}+\frac{L_{2}}{R_{2}}=\frac{R^{\prime} M_{1}}{R_{1} R_{2}}-\frac{\mathrm{I}}{\omega}\left(\tau_{1}+\tau_{2}\right)
\end{aligned}
$$

By using three shunts and measuring the three sums of the time constants the resulting independent equations may be solved for the time constants of the individual resistances.

This method differs from the mutual-inductance method, described above, in the fact that here the drop in a resistance is balanced against the secondary voltage of a mutual inductance, while in the former case the two shunts are balanced against each other. The result of this is that the balance is extremely sensitive to slight changes in frequency and the unsteadiness caused by fluctuations in the speed of the driving motor is the practical limit to the sensitivity of the arrangement. 
Both methods require a knowledge of the phase defects " $\tau$ " of the mutual inductances used. The values obtained in the preliminary work referred to above were based on certain values of $\tau$, which further investigation has shown to be incorrect. The results of a recomputation of the quarter-phase measurements using the new values of $\tau$ are given in Table 5. The four time

TABLE 5

Summary of Quarter-Phase Method

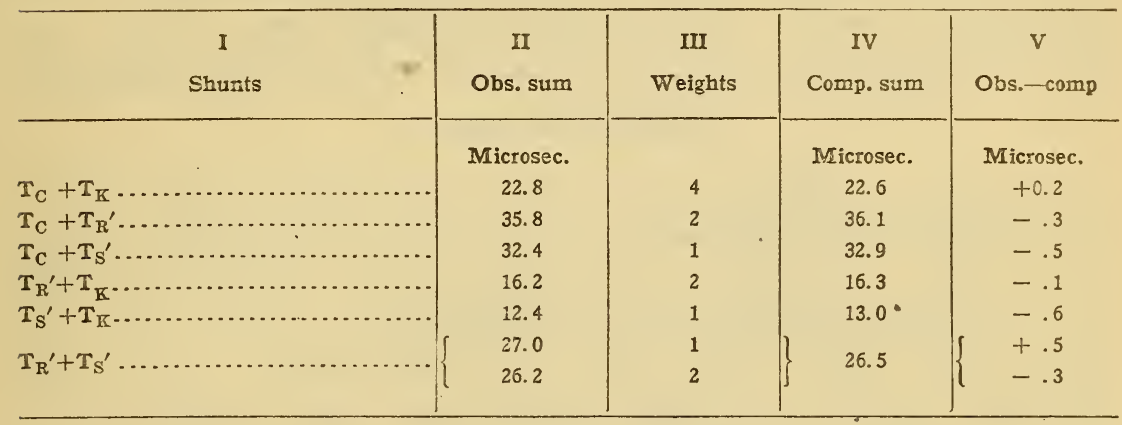

constants which best fitted the observed sums in column II were found and column IV computed from these values. The results are thus seen to be consistent to about 0.5 microsecond, which is probably better than could be normally expected without careful attention to speed control.

The shunts $S^{\prime}$ and $R^{\prime}$ are not comparable with $S$ and $R$ of the later work since they were modified in the arrangement of potential leads. $C$ and $K$, however, are the same, and values obtained by this quarter-phase method have been included in the final adjustment given below.

\section{CHANGE OF RESISTAINCE METHOD}

The second method for the direct measurement of inductance without recourse to computation is based upon the use of two shunts having the same inductance but different resistance. A measurement of the difference in time constant of two such shunts gives

$$
\frac{L}{R_{1}}-\frac{L}{R_{2}}=\Delta T
$$

or

$$
L=\Delta T \frac{R_{1} R_{2}}{R_{2}-R_{1}}
$$


The condition of equality of inductance can be obtained by constructing two shunts of identical dimensions, but of materials having different resistivity, or by using the same shunt at two temperatures (provided it has a high temperature coefficient of resistivity).

In the first case it is not essential that the dimensions be identical if the shape is such that the inductance can be computed, since the computed difference can be applied as a correction. That this correction does not invalidate the independence of the method is shown by the following reasoning: Let $L_{1}$ and $L_{2}$ be the true values of the inductance and $L_{1}{ }^{\prime}$ and $L_{2}{ }^{\prime}$ be the computed values. Substitution in (48) gives

$$
\begin{aligned}
& T_{1}=\frac{L_{1}}{R_{1}}=\frac{\Delta T R_{2}}{R_{2}-R_{1}(\mathrm{I}-\nu)} \\
& T_{2}=\frac{L_{2}}{R_{2}}=\frac{\Delta T R_{1}}{R_{2}-R_{1}(\mathrm{I}-\nu)}-\nu \frac{\Delta T R_{1}}{R_{2}-R_{1}(\mathrm{I}-\nu)}
\end{aligned}
$$

where

$$
\nu=\frac{L_{1}-L_{2}}{L_{1}}
$$

Now $\nu$ may be written

$$
\nu=\frac{\left(L_{1}-L_{1}{ }^{\prime}\right)-\left(L_{2}-L_{2}{ }^{\prime}\right)+\left(L_{1}{ }^{\prime}-L_{2}{ }^{\prime}\right)}{L_{1}}=\frac{\Delta_{1}-\Delta_{2}+\Delta_{3}}{L_{1}}
$$

Where $\Delta_{3}$ is the computed difference and is therefore known. The terms $\Delta_{1}$ and $\Delta_{2}$ are the errors in the individual computed values, and it is seen that it is only their difference which enters the correction term in (5I). This source of error is particularly small since the main sources of $\Delta_{1}$ and $\Delta_{2}$, i. e., end effects and mutual inductance in the potential circuit, would be the same in both cases.

Two such pairs of shunts were used in the present investigation, and the results of the measurements are given in Table 6 . It is seen that a comparatively large difference in the computed inductance of the standards produces only a very small correction term. 
TABLE 6

Results of Direct Determinations "by Construction"

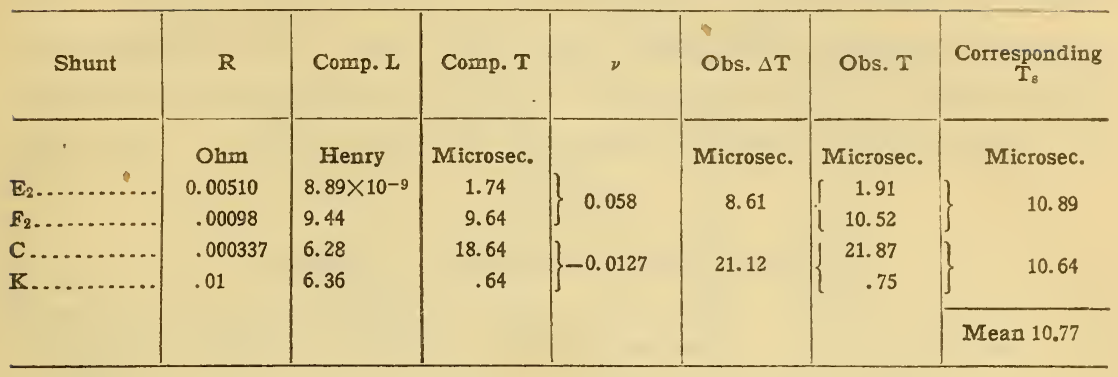

\section{HEATING METHOD}

The second modification of this method, namely, using a shunt of large temperature coefficient and measuring the inductance at two temperatures, would appear to be even freer from error since there can be no question of the practical identity of the dimensions. On the other hand, even with a copper resistance it is necessary to use a considerable temperature range, and thus introduce thermal expansion and possible distortion.

The procedure which was found most satisfactory was to carry the shunt through a closed cycle of temperature over as wide a range as possible and at various points to measure, by the currenttransformer method, the difference $\Delta T$ in time constant between the copper shunt and a reference shunt of manganin kept at a constant temperature. Since this method also gives the resistance of the unknown, a separate temperature or resistance measurement is not required.

At any point we have the relation

or

$$
\begin{gathered}
T_{\mathrm{c}}-T_{\mathrm{m}}=\Delta T \\
R_{\mathrm{c}} \Delta T=L_{\mathrm{o}}-T_{\mathrm{m}} R_{\mathrm{c}}
\end{gathered}
$$

hence, if $R_{\mathrm{c}} \Delta T$ is plotted against $R_{\mathrm{c}}$, the result should be a straight line of slope $T_{\mathrm{m}}$. Any curvature in the line is an indication of lack of constancy in $L_{\mathrm{c}}$.

The shape which is believed to be the freest from errors due to distortion, is the inner of two concentric tubes such as was used in shunt $T$. It has been shown that eccentricity of the tubes does not affect the inductance and the symmetry of the arrangement would tend to produce a symmetrical temperature gradient and 
current distribution. Shunt $P$ was constructed of this shape. Also another copper shunt $Q$ of the usual bifilar strip type was built, and this heating method was used with both types. The temperature range used was from $-50^{\circ} \mathrm{C}$ to $+150^{\circ} \mathrm{C}$, the lower temperatures being obtained in a bath of gasoline and $\mathrm{CO}_{2}$ snow. The results of the measurements are given in Table 7 . It is seen

TABLE 7

Results of Direct Determination by Heating

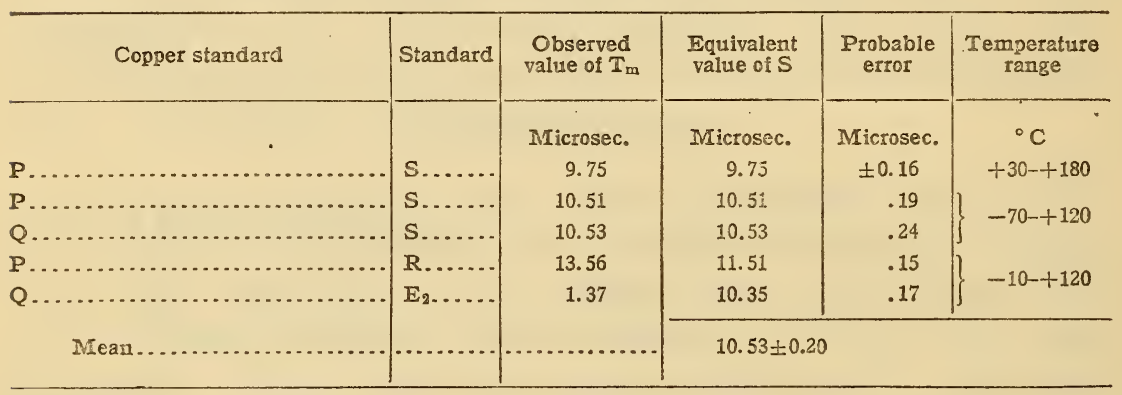

that the values vary considerably and much more than is indicated by the probable errors which were computed from the deviations of the individual points from the straight line for each run. This is probably due to changes in current distribution caused by local irregularities in temperature and hence resistance. This is the more surprising in that a longitudinal temperature gradient should produce no harm and the error is therefore probably due to variations over the cross section of the copper.

\section{ADJUSTMENT OF FINAL VALUE}

It is evident from the results quoted above that the relative measurements can be made with much greater accuracy than the direct determinations. The adjusted results of the difference measurements were therefore taken as correct, and the true magnitude of one of the shunts was assigned from a consideration of all the direct measurements.

The shunt $S$ was most centrally located in the network of differences, and all direct measurements have been referred to $S$ by the corresponding differences. The values resulting from the method of direct computation are tabulated in Table 8, the weights being assigned arbitrarily after a consideration of the probable constant errors of each type and the number and self-consistency of the observations involved. 
TABLE 8

Summary of Values by Computation

\begin{tabular}{|c|c|c|c|c|c|}
\hline Standard shunt & Type & $\begin{array}{l}\text { Inductance } \\
\text { computed }\end{array}$ & $\begin{array}{l}\text { Time constant } \\
\text { computed }\end{array}$ & $\begin{array}{c}\text { Corre- } \\
\text { sponding } T_{8}\end{array}$ & Weight \\
\hline $\mathbf{E}_{2} \ldots$ & Flat strip ...... & $\begin{array}{l}\text { Henrys } \\
8.89 \times 10^{-9} 9\end{array}$ & $\begin{array}{l}\text { Seconds } \\
1.74 \times 10^{-6}\end{array}$ & $\begin{array}{c}\text { Seconds } \\
10.72 \times 10^{-6}\end{array}$ & 8 \\
\hline$T_{23} \ldots \ldots \ldots$ & Tube........... & -0.47 & -0.46 & 10.75 & 8 \\
\hline $\mathbf{N}_{\mathrm{i}} \ldots \ldots \ldots \ldots$ & .....do.......... & -1.20 & -0.61 & 10.87 & 2 \\
\hline $\mathbf{A}_{2} \ldots \ldots$ & wire.......... & 247.0 & +2.47 & 10.61 & 2 \\
\hline Sec via $\mathbf{M} \ldots$ & Flat strip...... & 9.5 & 0.19 & 10.74 & 1 \\
\hline $\mathbf{S e c}$ via $\mathbf{A}_{2} \ldots \ldots \ldots$ & .....do......... & 17.0 & 0.17 & 10.57 & 1 \\
\hline $\mathrm{Me}$ & & & & $10.73 \pm .05$ & \\
\hline
\end{tabular}

The quarter-phase method gave $T_{c}=2 \mathrm{I} .2$ and $T_{k}=\mathrm{I} .4$ microseconds; hence we get $T_{\mathrm{S}}=10.0$ and II.3 microseconds, respectively. The results of the heating method are taken from Table 7 and those marked "construction" from Table 6. The comparison with the I-ohm standard referred to on page - gave $T_{S}=\mathrm{ro} .68$ microseconds. Assigning weights according to the self-consistency of each method, we get from Table 9 the value $T_{S}=10.7$ microseconds as the most probable basis for the system. The values of the other shunts follow directly from the difference measurements and are given in Table 2.

TABIE 9

Summary of Final Values

\begin{tabular}{|c|c|c|c|}
\hline Method & $\mathbf{T}_{\mathrm{s}}$ & Ave. dev. & Weighas \\
\hline Computation............ & $\begin{array}{c}\text { Microsec. } \\
10.73\end{array}$ & $\begin{array}{c}\text { Microsec. } \\
\pm 0.05\end{array}$ & 20 \\
\hline Quarter-phase................ & 10.6 & $\pm \cdot 5$ & 1 \\
\hline Heating ................... & 10.53 & \pm .2 & 1 \\
\hline 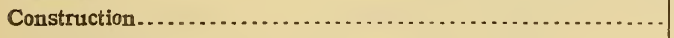 & 10.77 & \pm .09 & 5 \\
\hline 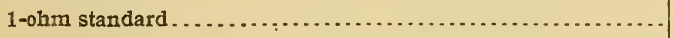 & 10.68 & $\pm \cdot 1$ & 5 \\
\hline 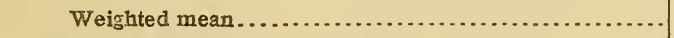 & 10.72 & \pm .03 & 32 \\
\hline
\end{tabular}

It is believed that these values represent the absolute time constants of the various shunts to within 0.1 or 0.2 microsecond and the relative values with rather greater precision. These standards having once been established, measurements on other shunts may be made easily by relative measurements by the current transformer or some similar method. 


\section{EFFECT OF STRAY FIELDS}

A factor which is often neglected in the design of shunts for use on alternating currents, but which is worthy of more attention, is the influence of stray fields. An ideal piece of apparatus should not be affected by the presence of stray electromagnetic fields, nor should it produce any field itself at outside points. In general, a design which reduces one of these effects reduces the other also; but this is not necessarily the case. The effect can be considered as a mutual inductance between the shunt and the rest of the circuit $S$. The error introduced into the inductance of the shunt is $M_{s p}$, i. e., the mutual inductance of the "potential circuit" $P$ and the surroundings $S$. The effect on the surroundings, however, is $M_{s c}$, i. e., the mutual inductance between the "current circuit" and the surroundings. In a shunt of Type I these values coincide, but this is not the case in Type II. One great advantage of some of the tubular types of shunt is that they satisfy this ideal condition very closely.

In comparing the behavior of different shunts in this respect it is necessary to have some standard stray field as a basis of reference. I have taken for this a uniform field of intensity $H=I O I$, where $I$ is the current in the shunt in amperes. This field is much larger than is likely to occur unintentionally in practice, but can easily be approximately realized experimentally. Since the effect depends on the direction of the field, that direction giving the maximum effect is the most definite and should be taken. In cases where the proper direction is not evident from the shape of the conductor, the maximum effect may be found as the resultant of the three components observed with fields along any three mutually perpendicular axes.

TABLE 10

Effect of Stray Fields

\begin{tabular}{|c|c|c|c|c|}
\hline Shunt & Resistance & $\mathbf{T}$ & $\Delta \mathbf{T}$ & $\frac{\Delta R}{R}$ \\
\hline & Ohm & Microsecs & Microsecs & \\
\hline $\mathbf{N}_{x} \ldots \ldots \ldots \ldots \ldots$ & 0.0026 & .......... & 0.9 & \\
\hline $\mathrm{T}_{13} \ldots \ldots \ldots$ & .002 & -0.5 & .3 & ..... \\
\hline $\mathrm{E}_{2} \ldots \ldots \ldots \ldots$ & .005 & +1.7 & .8 & ............. \\
\hline L \& N $\ldots \ldots \ldots \ldots \ldots$ & .01 & 10.7 & 3.6 & (............. \\
\hline $\mathrm{D} \& \mathrm{~T} \ldots \ldots \ldots \ldots \ldots$ & .01 & 9.4 & 1.7 & $1.5 \cdot 10^{-4}$ \\
\hline 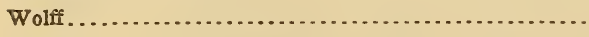 & .01 & 5.5 & 5.2 & 6.6 \\
\hline R & .0010 & 12.8 & 11.5 & 13.8 \\
\hline ( & .001 & 3.5 & 12.7 & 2.3 \\
\hline GE compensated $\ldots \ldots \ldots \ldots . . . . . . . . . . .$. & .001 & 0.1 & 5.0 & ....... \\
\hline
\end{tabular}

Table ro gives a summary of the effects observed on a variety of shunts, $\Delta T$ being the change in apparent time constant due to 
the presence of a standard field in the most effective direction. These results indicate that the effect is comparatively small in shunts in which the potential leads are kept close together even though the working material has fairly wide spacing as in the case of the $L \& N$ o.or ohm, where the spacing is one-sixth the width of the strips. In the last three cases, however, there are fairly large loops in the potential leads and a correspondingly large error from stray fields.

In making these measurements it was noticed that in some cases the shunts showed a change of resistance when in the magnetic field. This change reversed its sign with a reversal of the field and was traced to eddy currents in the metal case surrounding the shunt. The field due to these eddy currents is nearly in quadrature with that from the main current, and the emf induced by it is, therefore, in phase with the current and produces an apparent change in resistance.

Since most shunts are arranged with metal cases to contain oil for cooling, this source of error should be carefully guarded against even in work where the phase-angle does not enter. The changes in resistance expressed as a fraction of the nominal resistance, observed with the various types when in a field of standard intensity, are given in Table Io, in the last column.

\section{OBSERVATIONS ON VARIOUS TYPES OF SHUNT}

As wide a variety as possible of the alternating-current shunts now available were compared by the current-transformer method with the standards described above. The results are given in Table ı. The values of time constant are also plotted in Fig. II

TABLE 11

Summary of Results on Various Types of Shunts

\begin{tabular}{|c|c|c|c|c|c|c|}
\hline Shunt & Resistance & $\begin{array}{l}\text { Current } \\
\text { capacity }\end{array}$ & $x$ & $\mathrm{~T}$ & Remarks & \\
\hline & Ohms & Amperes & Henrys & Microsec. & \multirow{7}{*}{ For comparator. } & \\
\hline G. E. No. $182045 \ldots \ldots \ldots$ & 0.0025 & 200 & $5.21 \times 10^{-9}$ & 2.08 & & \\
\hline G. E. No. $182046 \ldots \ldots \ldots$ & .001 & 500 & 3.46 & 3.46 & & \\
\hline I \& N No. $13555 \ldots . . . \ldots .$. & .05 & 12 & 130 & 2.5 & & \\
\hline L \& N No. $13582 \ldots . . . \ldots .$. & .01 & 50 & 107 & 10.7 & & \\
\hline Drysdale D $\mathbf{D} . \ldots \ldots \ldots$ & .01 & 100 & 94 & 9.4 & & \\
\hline Drysdale auxiliary leads $D^{1}$ & .00989 & 100 & 20.6 & 2.1 & & \\
\hline Wolff No. $4361 \ldots . . . \ldots \ldots$ & .10 & ..... & 450 & 4.5 & \multirow{6}{*}{$\begin{array}{l}\text { Return at } \infty \text {. } \\
\quad \text { Do. } \\
\text { Return across top } \\
\text { Return at } \infty \text {. } \\
\text { Return across top }\end{array}$} & \\
\hline Wolff No. $4433 . . . . . . . .$. & .01 & 50 & 55 & 5.5 & & \\
\hline Do............. & $\cdots$ & . & 68 & 6.8 & & \\
\hline Woiff No.4481........ & .001 & 500 & 39.1 & 39.1 & & \\
\hline Do............ & & & 35.8 & 35.8 & & \\
\hline Paul No. 136........ & .10 & 15 & 410 & 4.1 & & \\
\hline
\end{tabular}


as ordinates with $\log _{10} R$ as abscissae. As might be expected, shunts of the same type fall along fairly smooth curves. For comparison the values published by Orlich and Paterson and Rayner are included.

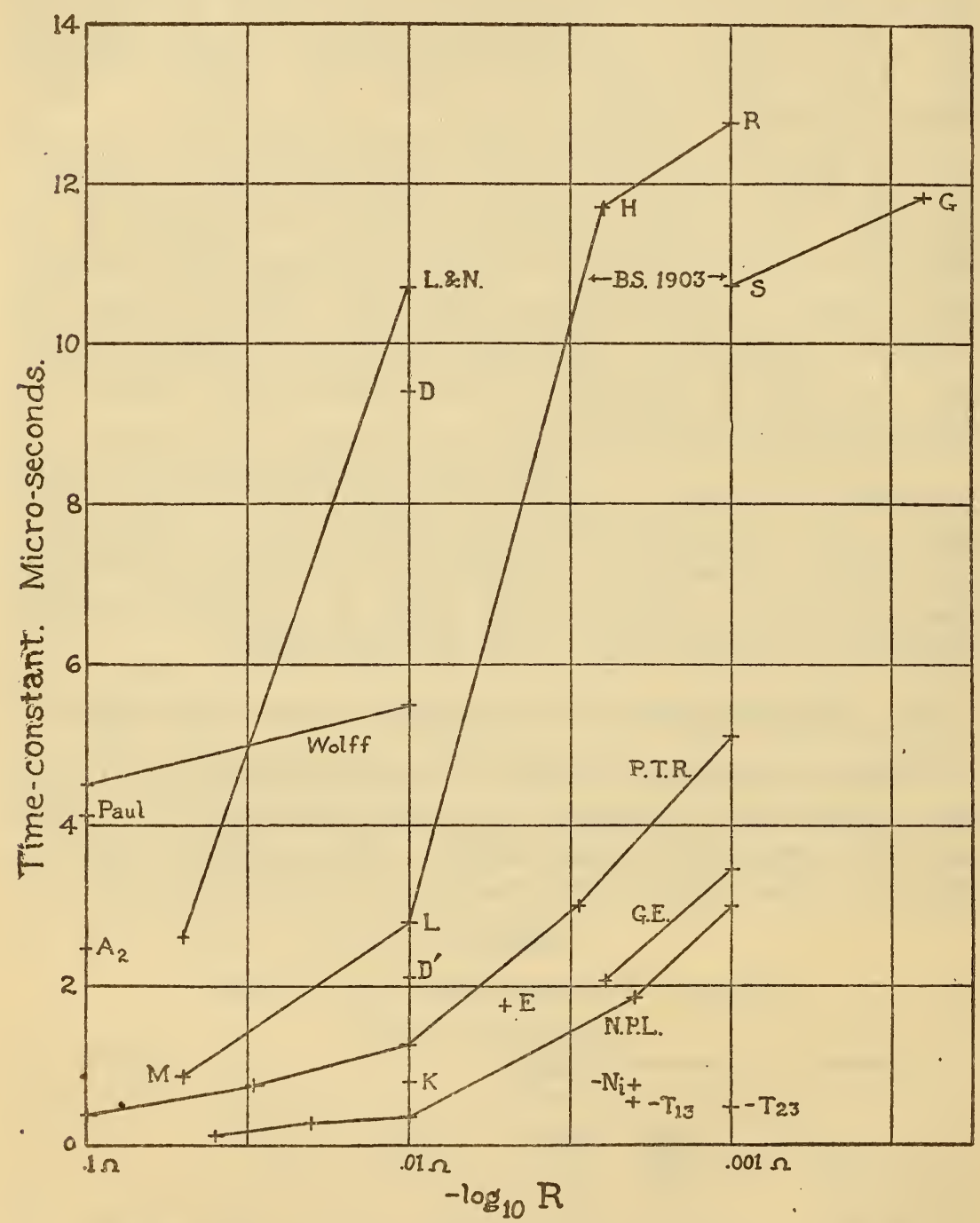

FIG. II.-Time constants of various types of shunts

It is seen that in each series the time constant increases as the resistance decreases. The logical basis for the design of a series of shunts would be a constant volt drop and constant energy dissipation per unit area of cooling surface. If it is further assumed (as is approximately the case in practice) that the cross section 
remains geometrically similar to itself, one would expect the time constant to vary as $R^{-\frac{4}{3}}$. The curves shown, however, correspond to a variation as the -0.7 power of the resistance. This is due to the fact that in the practical designs the lower resistance shunts are worked much nearer the limit of cooling.

The point $D$ corresponds to a o.or ohm shunt intended for alternating-current work. This is seen to have a much higher inductance than was to be expected. An investigation showed that 80 per cent of the inductance was in the copper rods leading down to the shunt proper, and point $D^{\prime}$ represents the result using potential leads attached directly to the resistance material.

\section{NOTES ON DESIGN}

In the design of shunts for use on alternating current one must consider not only the questions of permanency and heat dissipation as in direct-current shunts, but also the additional problems of low inductance and freedom from stray fields.

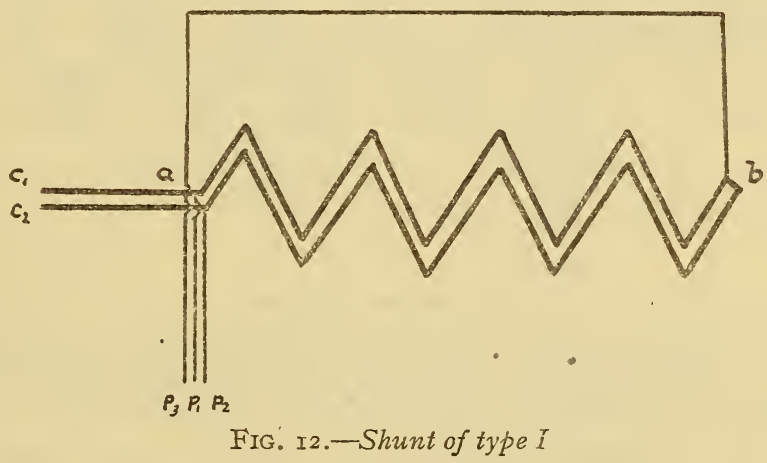

The problem of inductance has hitherto been attacked along two lines. In the first, as was done by Orlich, ${ }^{26}$ Drysdale, ${ }^{27}$ and others, the shunt is of type I, the working material is formed into a closed circuit of as little self-inductance as possible and the potential leads kept free from all inductive effects. In the second method, indicated by Lichtenstein ${ }^{28}$ and definitely suggested for shunts by Campbell, ${ }^{29}$ the potential leads are placed as close as possible to this working resistance so that the mutual inductance of the lead and the resistance is nearly equal and opposite to the self-inductance of the resistance. Figs. I2 and I3 represent

\footnotetext{
${ }^{26}$ Orlich, Zs. für Inst'k'de, 29, p. 24I; I909.

${ }^{27}$ Drysdale, Elec'n, 66, p. 34r; igro.

${ }^{28}$ Lichtenstein, Dinglers Polytchn. Jour., 321, p. I00; 1906.

${ }^{29}$ Campbell, Elec'n, 61, p. roo०; I908.
} 
schematically the two types. A notable example of the second type is the series described by Paterson and Rayner.

The time constant in Fig. I 2 is the same except for the effects of stray fields whether lead $p_{2}$ or $p_{3}$ is used. Now, if in Fig. I3 $p_{1}$ and $p_{2}$ are used for current terminals and $c_{1}$ and $c_{2}$ for potential terminals, the two types become identical. Now, by the reciprocal theorem ${ }^{30}$ the inductance of the arrangement in Fig. I3 is the same whether $p_{1} p_{2}$ are used as potential leads and $c_{1}$ and $c_{2}$ as current leads, or vice versa. It follows, therefore, that the inductance in either case is that of the strip $a b$ with its return either at $b c_{2}$ or $b p_{2}$. Hence the advantage of the second type lies solely in the possibility of making the potential lead $b p_{2}$ thinner than the current lead $b c_{2}$. In the limiting case of zero thickness for both $b p_{2}$ and the insulation, the maximum gain is only a factor one-half.

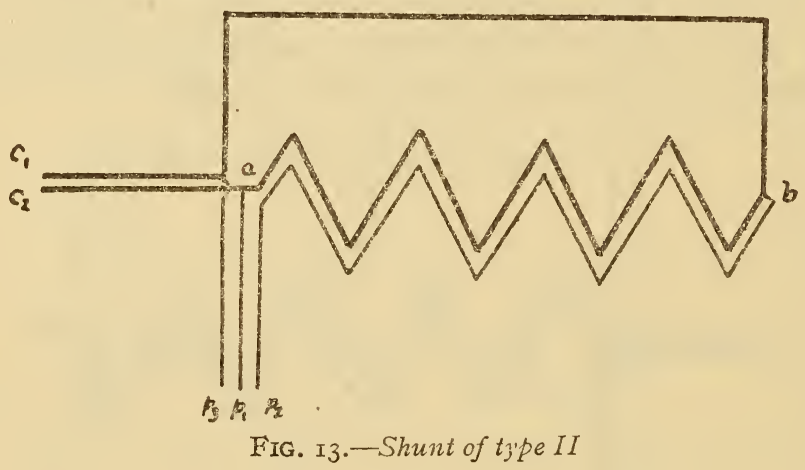

Of course, any increase in the rate of cooling permits of a decrease in the amount of working material, and consequently in the time constant. The General Electric Co., by the very ingenious use of large copper vanes (which carry no current), have been able to obtain a large energy dissipation with a small amount of manganin. The shunts described by Paterson and Rayner also have a very effective cooling, but in this case it has been employed to raise the permissible volt drop, and only a little of the latitude obtained by the cooling has been devoted to reducing the inductance. It is clear that with the spacing at all close either of these types practically sacrifices one-half the cooling surface of the metal. All of these types, moreover, tend to greater time constants with decreasing resistance.

Drysdale has proposed a type in which the lower resistances are built up of a number of units in parallel. With such an arrange- 
ment the phase angle would be independent of resistance. $\mathrm{He}$ estimates a time constant of $1.03 \times 10^{-6}$ second to be easily attainable. ${ }^{31}$ Sharp and Crawford mention a similar design, which, however, had $T=I 7 \cdot 10^{-6}$ second.

The method of attack which seems most promising to the author is to design the shunt satisfactorily from the cooling point of view and then insert in one of the potential leads sufficient mutual inductance to balance exactly the self-inductance of the manganin between the potential points. This can, of course, always be done by a small coil placed near the heavy-current leads. The most serious objection to this practice is the danger of stray fields, but a number of devices can be used which would render this adjusting coil nearly astatic. This method, of course, requires a measurement of the inductance, but that would be necessary anyway and with the convenience of the currenttransformer method would not be a hardship.

The tubular arrangement has many advantages and permits of some rather neat schemes for adjustment. If the inner conductor be used as the working resistance and the potential leads be brought out inside the inner tube (see Fig. $9 d$ ) as was done with shunts $T$ and $N_{i}$, then the mutual-inductive effects are too great and the shunt shows a negative inductance. This excess is not very large, however, and even in a o.oor-ohm standard could be neglected in almost all work. ${ }^{32}$

This negative excess can be reduced to any desired amount by the arrangement shown in Fig. I4 (a). For an exact balance the distances $l$ and $l^{\prime}$ should be in the ratio given by-

$$
\begin{gathered}
l\left[\frac{a_{2}^{2}+a_{1}^{2}}{2\left(a_{2}^{2}-a_{1}^{2}\right)}-\frac{2 a_{1}^{2} a_{2}^{2}}{\left(a_{2}^{2}-a_{1}^{2}\right)^{2}} \log \frac{a_{2}}{a_{1}}\right]=l^{\prime}\left[2 \log \frac{a_{3}}{a_{2}}+\right. \\
\left.\frac{2 a_{4}^{2}}{a_{4}{ }^{2}-a_{3}^{2}} \log \frac{a_{4}}{a_{3}}+\frac{2 a_{1}^{4}}{\left(a_{2}{ }^{2}-a_{1}^{2}\right)^{2}} \log \frac{a_{2}}{a_{1}}-\frac{a_{2}{ }^{2}+a_{1}{ }^{2}}{2\left(a_{2}{ }^{2}-a_{1}{ }^{2}\right)}\right]
\end{gathered}
$$

or approximately $\quad \frac{l}{l^{\prime}}=2+3 \frac{s}{t}+6 \frac{u}{t} \cdots$

where as before $s=\frac{a_{4}-a_{3}}{a_{4}}, t=\frac{a_{2}-a_{1}}{a_{2}}, u=\frac{a_{3}-a_{2}}{a_{3}}$.

${ }^{31}$ The author regrets that he has been unable to obtain a shunt of this type to measure. The one listed in the table consisted of a single strip.

32 Using this arrangement with one of the N. P. $I$. tubes would give $L=-I \times 10^{-9}$ henry instead of $+3 \times 10^{-9}$ henry as now arranged. 
Another interesting combination is that shown in Fig. I4 (b). Here (with suitable ratios of $l$ to $l^{\prime}$ ) we have a shunt having zero phase angle, and also no emf induced in the potential leads. In other words, the negative inductance of the outer tube with return inside is balanced by the positive inductance of the shorter length of inner tube so that the difference in potential between the points $a$ and $b$ is in phase with the total current. If the inductance of the disk is neglected, the ratio should be

$$
\frac{l}{l^{\prime}}=3+2 \frac{t}{s}+6 \frac{u}{s} \text { (approx.) }
$$
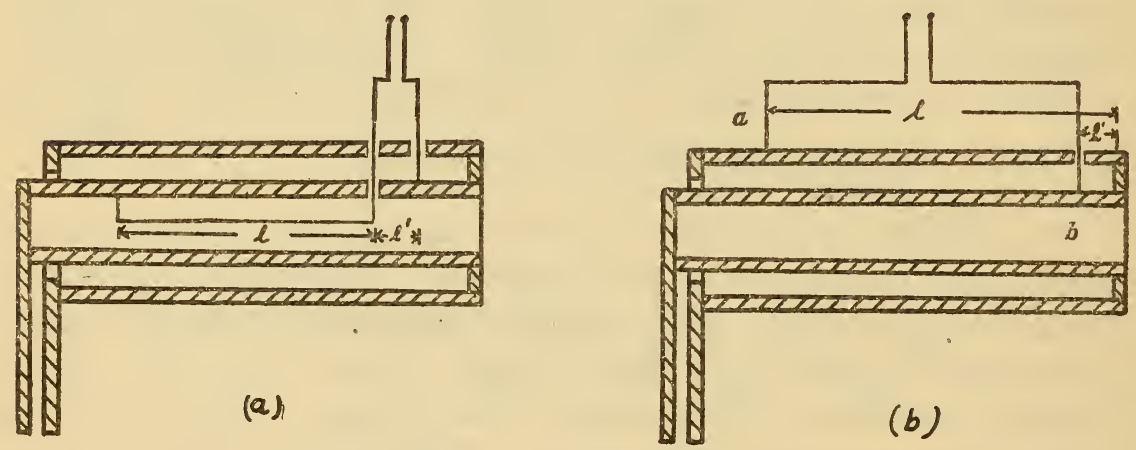

FIG. I4.-Tubular shunts with compensating potential leads

\section{MUTUAL INDUCTANCES}

A mutual inductance has usually been regarded as yielding a secondary emf in exact quadrature with the primary current, though occasionally the necessity of using stranded wire in the windings has been pointed out. In case the primary is to carry a heavy current the departure from quadrature may become very considerable. Using as primary a copper strip $5 \mathrm{~cm}$ by $0.32 \mathrm{~cm}$ two secondary coils showed voltages having a phase difference of 75 minutes at 60 cycles per second. With due care, however, the phase defect of a mutual inductance may be made much smaller than that of a shunt of the same current capacity, so that the mutual inductance and quarter-phase methods, described above, are most useful in measuring the shunts, rather than in using the shunts to measure the mutual inductances.

The factors which produce this shift of phase can be seen by working out in detail the case where the primary and secondary windings each consist of two thin wires in parallel as indicated in 
Fig. I5. Applying Kirchoff's laws to these four circuits, we get for the relation between secondary voltage and primary current

$$
E_{s}=I_{p}(R+j \omega M)
$$

where $M=M_{24}+\frac{I}{R_{s} R_{p}}\left[M_{13} R_{2} R_{4}+M_{14} R_{2} R_{3}+M_{23} R_{1} R_{4}\right.$

$$
\left.-M_{24}\left(R_{2} R_{4}+R_{2} R_{3}+R_{1} R_{4}\right)\right]
$$

and

$$
\begin{aligned}
R= & \frac{\omega^{2} R_{1} R_{2} R_{3} R_{4}}{R_{p} R_{s}}\left\{\frac{\mathrm{I}}{R_{s}}\left(\frac{L_{4}{ }^{\prime}}{R_{4}}-\frac{L_{3}{ }^{\prime}}{R_{3}}\right)\left(\frac{A}{R_{1}}+\frac{B}{R_{2}}\right)\right. \\
& \left.+\frac{\mathrm{I}}{R_{p}}\left(\frac{L_{2}^{\prime}}{R_{2}}-\frac{L_{1}^{\prime}}{R_{1}}\right)\left(\frac{C}{R_{s}}+\frac{D}{R_{4}}\right)\right\}
\end{aligned}
$$
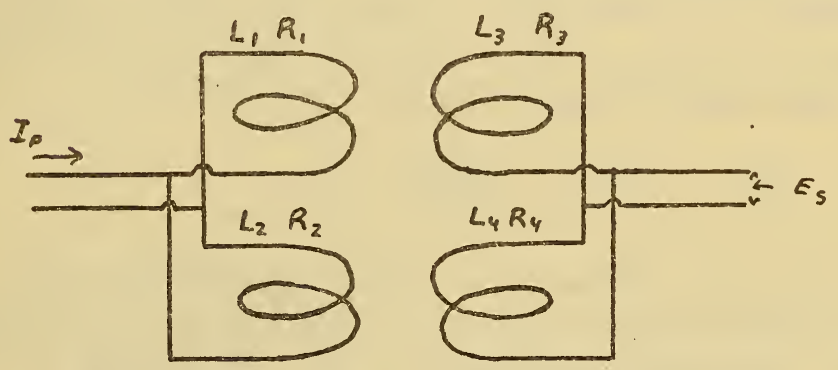

FIG. 15.-Four-coil model of impure mutual inductance

In these equations $R_{1}$ and $L_{1}$ are the resistance and inductance of the circuit I, $R_{2}$ and $L_{2}$ of circuit 2, etc., and $M_{12}$ is the mutual inductance between circuits I and 2, etc.

$$
\begin{aligned}
& \text { Also } L^{\prime}{ }_{1}=L_{1}-M_{12}, L_{2}^{\prime}=L_{2}-M_{12}, L_{3}{ }^{\prime}=L_{3}-M_{34}, L^{\prime}{ }_{4}=L_{4}-M_{34} \\
& R_{p}=R_{1}+R_{2}, R_{s}=R_{3}+R_{4} \\
& A=M_{13}-M_{14} \\
& B=M_{23}-M_{24} \\
& \text { and } \quad \begin{array}{c}
C=M_{13}-M_{23} \\
D=M_{14}-M_{24}
\end{array}
\end{aligned}
$$

In deriving these equations terms of higher order in $\frac{\omega L}{R}$ and $\frac{\omega M}{R}$ have been neglected. An inspection of (59) shows that this resistance term is in two parts, the first of which may be considered as due to the secondary circuit, while the second term in the brackets is due to the primary circuit. This can be seen most clearly by letting one (say, the secondary) circuit become linear, i. e., let 3 and 4 coincide. Then the first term vanishes but the 
second term remains and is a function of the dimensions and relative position of the parts of the primary circuits. For brevity these terms will be referred to as the "secondary impurity" and "primary impurity," respectively.

It will also be noticed that each of these terms contains two factors. The first $\frac{L_{2}^{\prime}}{R_{2}}-\frac{L_{1}^{\prime}}{R_{1}}$ is proportional to the difference in phase of the two parts of the primary current. The second involves $\left(M_{13}-M_{23}\right)$ and $\left(M_{14}-M_{24}\right)$, that is, the difference in the mutual inductance of various parts of the primary on a single part of the secondary. If either factor vanishes, then the whole term will vanish regardless of the other factor.

The deduction of the equation (59) may be generalized to cover any number of elementary filaments and thus to represent a filamentary flow in a solid conductor. In case the secondary is linear the primary impurity becomes proportional to

$$
\sum_{\mathrm{k}=\mathrm{I}}^{\mathrm{k}=\mathrm{n}} \Delta \theta_{\mathrm{k}} \Delta m_{\mathrm{k}}
$$

Where $\Delta \theta_{\mathrm{k}}$ is the difference in phase angle between the current in the $k$ th filament and the total current and $\Delta m_{\mathbf{k}}$ is the difference between the mutual inductance of the $k$ th filament on the secondary and the average mutual inductance. The expression for the secondary impurity is of course similar.

The usual method of stranding tends to reduce both factors in these errors, but becomes very laborious if the coil is to carry heavy currents. $A$ and $B$ of (59) become zero, regardless of the thickness of the secondary wire if the magnetic field of the primary is so localized that there is no magnetic field at points where the secondary copper is placed. Conversely $C$ and $D$ will vanish if a current in the secondary produces no magnetic field where the primary copper is located. The required localization of the magnetic field to make either pair zero is easily obtained by using one winding in the form of a uniformly wound toroid, or a long straight solenoid (which is really a special case of the former). It is, however, impossible to make both errors zero by this arrangement, since if both circuits are closed toroids they either have no flux in common or one is within the other and its copper is in the field of the other.

The logical way to construct a pure mutual inductance is, therefore, to eliminate one source of impurity by making $A$ and $B$ 
zero and to minimize the other by making the time constant of the various elementary filaments as nearly equal as possible. This means making one coil in the form of a uniformly wound toroid and then stranding this coil. To be efficient the strands should, of course, be thoroughly intermixed. With such a construction the character of the other coil is immaterial. It is therefore preferable to use the toroidal coil as secondary, since one can then use as heavy copper as desired in the primary. A coil of this type used in this work showed no detectible primary impurity, though the primary winding was of 130000 circular mil cable.

Equation (6o) forms the basis of a method of testing inductances having stranded primary windings. Consider a coil having a linear secondary and the primary wound from a number of parallel strands with a small coil $c_{1}, c_{2} \ldots$ in series with each strand. Using each strand separately as a primary, the mutual inductance between it and the secondary can be measured. Subtracting each of these values from the average of all gives the successive values of $\Delta m_{k}$. Now, passing the full primary current through all the strands in parallel the

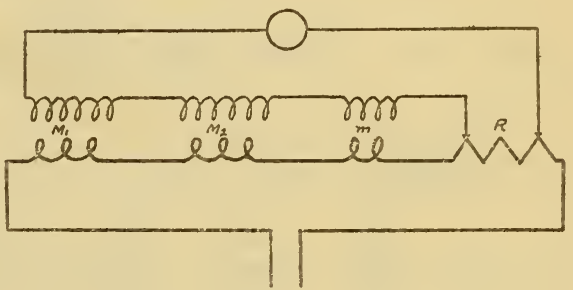

FIG. I6.-Connection for comparing the phase defects of mutual inductances relative phases of the components are to be determined. This can be done by placing a small exploring coil near each of the coils, $c_{1}, c_{2} \ldots$ and observing the phase of the induced emf relative to any fixed standard. The differences of these values from their mean give the values of $\Delta \theta_{k^{\circ}}$. The summation of (60) may thus be directly observed and gives the amount by which the primary impurity differs from that due to a single strand. This method was used in one of the mutual inductances used in the resistance measurements where there were I2 strands and was found very convenient.

What is perhaps the simplest method of comparing the phase defects of mutual inductances of nearly equal magnitude is indicated in Fig. I6. The two inductances to be compared are connected with their primaries in series with the primary of a small variable mutual inductance $m$ and with a very low resistance $R$. This latter may well be arranged as in Fig. 6 . The secondaries of $M_{1}$ and $M_{2}$ are connected in opposition and any difference in voltage is balanced by $m$ and $R$. At a balance the algebraic sum of the $67154^{\circ}-$ vol $13-16-5$ 
three mutual inductances and also of the four four-terminal "resistances" (as defined by equation 9, page 38I) must be zero. By a little ingenuity in connecting coils in series, it is possible to intercompare mutual inductances of widely different values without ever using a very large variable inductance at $m$. The "resistance" of $m$ may therefore be kept negligible.

While the primary error is the more dangerous, the secondary error may not be negligible. A number of measurements were made on circuits in which the primary error had been eliminated by using a toroidal or solenoidal secondary. These comparisons brought out some interesting facts about this secondary error.

TABLE 15

Results of Measurements on Secondary Impurity of Mutual Inductances

\begin{tabular}{|c|c|c|c|c|c|c|c|c|}
\hline Coil & M & $\begin{array}{l}\text { Radius of } \\
\text { coil }=r\end{array}$ & $\begin{array}{c}\text { Radius of } \\
\text { wire }=a\end{array}$ & $\begin{array}{c}\text { Turns } \\
\text { per } \\
\text { centi- } \\
\text { meter } \\
\text { n }\end{array}$ & $a^{4} n^{2} r$ & $\mathbf{T}$ & $\frac{\mathbf{R}}{\mathrm{f}^{2}}$ & Remarks \\
\hline p.. & $\begin{array}{l}\text { Micro- } \\
\text { henrys } \\
4.40\end{array}$ & ${ }_{1.32}^{\mathrm{cm}}$ & $\begin{array}{l}\mathrm{cm} \\
0.020\end{array}$ & 7.5 & $\begin{array}{c}\mathrm{cm}^{3} \\
0.14 .10-4\end{array}$ & $\begin{array}{c}\text { Micro- } \\
\text { seconds } \\
0.03\end{array}$ & $\begin{array}{l}\text { Ohms per } \\
\text { cycle }^{2} \\
+6 . \times 10^{-12}\end{array}$ & Solenoid. \\
\hline $1 .$. & .47 & .60 & .040 & 4.2 & .27 & .39 & +7.0 & Do. \\
\hline m. & 6.00 & 1.05 & .020 & 16.0 & .54 & .00 & -0.0 & Do. \\
\hline 1a.. & 4.89 & 1.14 & .032 & 8.1 & .75 & .04 & +8.0 & Toroid. \\
\hline $0 \ldots$. & 1.18 & .60 & .040 & 9.5 & 1.41 & .23 & 11.0 & Solenoid. \\
\hline $5 g_{1}$. & 3.23 & $* 2.64 \times 3.00$ & .040 & 7.79 & 2.23 & .14 & 18.0 & Toroid. \\
\hline $\mathbf{h}_{1} \ldots$ & 5.25 & 1.25 & .040 & 9.5 & 2.90 & .10 & 20.0 & Solenoid. \\
\hline n.... & 2,90 & 1.07 & .051 & 7.5 & 4.03 & .34 & 39.0 & Do. \\
\hline f.... & 4.89 & 1.38 & .051 & 7.3 & 4.98 & .22 & 43.0 & Do. \\
\hline q... & 3.50 & 1.39 & $\dagger 7 \times 0.01$ & 5.2 & $\ldots$. & .06 & 8.0 & $\begin{array}{c}\text { Litzen-draht } 5 \times 7 \\
\text { No. } 35 \text { B. \& S. }\end{array}$ \\
\hline $\mathbf{h}_{2} \ldots$ & 5.97 & 1.33 & .040 & 9.5 & 3.09 & .29 & 69.0 & Outside $h_{1}$. \\
\hline$h_{3} \ldots$ & 6.73 & 1.43 & .040 & 9.5 & 3.30 & .43 & 115.0 & Outside $h_{2}$. \\
\hline $\mathbf{h}_{4} \ldots$ & 7.58 & 1.51 & .040 & 9.5 & 3.48 & .61 & 182.0 & Outside $\mathbf{h}_{3}$. \\
\hline $\mathbf{h}_{5} \ldots$ & 8.38 & 1.60 & .040 & 9.5 & 3.69 & .73 & 242.0 & Outside $\mathrm{h}_{4}$. \\
\hline $5 g_{2} \ldots$ & 3.66 & $* 2.87 \times 3.27$ & .040 & 7.58 & 2.59 & .20 & 29.0 & $\left\{\begin{array}{l}\text { Toroid. } \\
\text { Outside gl. }\end{array}\right.$ \\
\hline
\end{tabular}

* Coils $g_{1}$ and $g_{2}$ were of rectangular section and of the dimensions indicated.

$\dagger$ Each coil $q$ consisted of one of the five seven-wire strands,

The group of 15 coils compared in this manner are listed in Table I5. The complete mathematical calculation of the error due to eddy currents in the secondary would be extremely difficult, even in the simplest case of a long straight uniform solenoid. A rough approximation may be obtained for this case by assuming the actual secondary wire replaced by two thin filaments parallel to the axis of the wire and at a distance apart $2 y$ in the direction of the radius of the coil. It may also be assumed that the ordinary 
two-terminal resistance of each filament is equal to twice the resistance of the complete wire. The various quantities entering equation (59) may then be computed and it appears that the "resistance" is given by

where

$$
R=K \omega^{2} a^{2} n^{2} y^{2} r
$$

$\omega=2 \pi \times$ frequency, $a=$ radius of wire.

$n=$ turns per centimeter of winding, $r=$ radius of coil.

In case $y$ may be taken a constant fraction of $a$ as would appear reasonable if $r$ is large, we have

$$
R=K^{\prime} \omega^{2} a^{4} n^{2} \gamma
$$

This is in agreement with dimensional reasoning, which shows that $R$ must be reducible to the form

$$
R=\omega a \psi\left[\frac{\omega a^{2}}{\rho}, \text { an }, \frac{a}{r}\right]
$$

where $\rho$ is the resistivity of the material and $\psi$ an unknown function.

The observed differences of $\frac{R}{f^{2}}$ for the various coils from an arbitrary zero have been plotted in Fig. I $7(I)$ against the quantity $a^{4} n^{2} r$. According to (62) this should yield a straight line. The points seem to indicate a slight curvature, but the agreement is within the precision needed in the work on shunts. An extrapolation of the line to the axis where $a^{4} n^{2} r=0$ gives the true zero for the system. It may be noted that points corresponding to the closed toroids $5 g_{1}$ and $\mathrm{I} a$ fall along the same line as those for the long straight solenoids.

In coils of more than one layer, the outer layers show an abnormally great secondary error. This is shown in Fig. I 7 (II), where the time constants of five coils wound one above the other are plotted against the values of $a^{4} n^{2} r$. The innermost coil is seen to be normal, while the outer coils have a greatly increased phase defect. This effect is due to the fact that the eddy currents in the inner wires produce a magnetic field in quadrature with the main field. This field is linked with all the coils outside of it and induces a large out-of-phase emf in them, but does not link the coils inside of it. This shows the danger of using multiple layer coils for work of this character. 
A further source of secondary impurity is the capacity of the windings. If a condenser of capacity $C$ is connected across the terminals of a secondary winding of resistance $R$, then the mutual inductance will show an apparent phase defect of

$$
\tau=\omega c R
$$

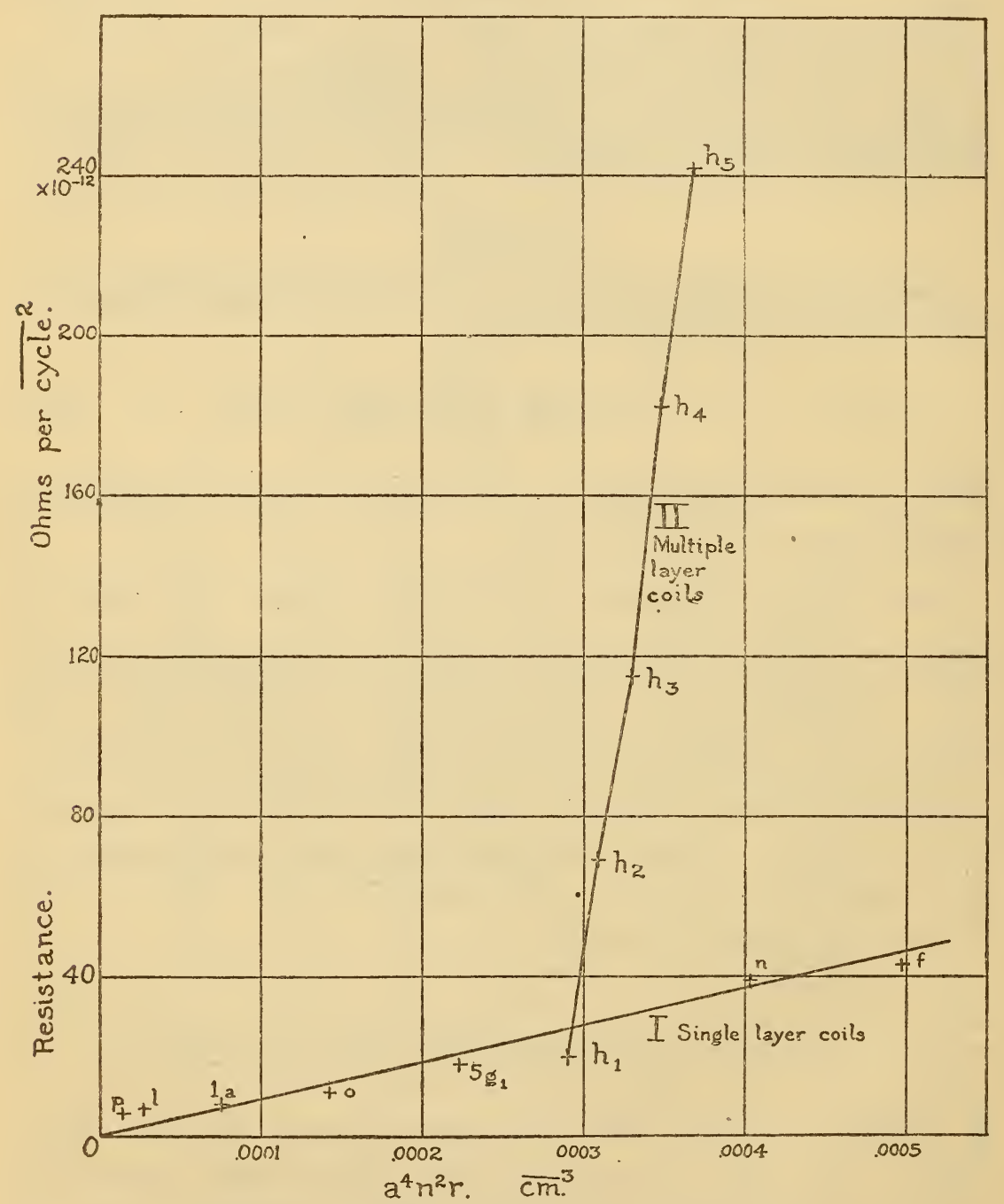

FIG. 17.-Secondary impurity of muinal inductances

This effect is entirely negligible in coils of the range used, say $M=\mathrm{IO}^{-5}$ henry, but may become very serious when the resistance of the windings becomes large. In comparing the phase angles of mutual inductances, this effect of capacity is useful, 
since by connecting a microfarad across one of the coils, its emf is made to lag more than before, and by noting the direction of the change in the resistance setting, it is readily seen which coil has the greater phase angle.

\section{SUMMARY}

The results of this study of four-terminal impedances are summarized below. The discussion of the inductance of shunts has been clarified by the classification of such apparatus into two main types and also by the use of the idea of the inductance of an open circuit "with a definite return." The close analogy between shunts and mutual inductances has been pointed out. The causes of phase defects in mutual inductances have been pointed out, and methods of avoiding or minimizing these sources of error suggested. The secondary impurity has been investigated and the abnormal increase of this error in multiple layer coils noted.

On the more practical side two relative methods for the comparison of the time constants of shunts have been studied and the sources of error determined. One of these has been shown to be very simple, accurate, and easily set up. Three possible types of shunts whose inductance can be computed from the dimensions have been studied, and the sources of error shown to be much smaller than would be expected. The formulæ for various arrangements of tubular shunts have been deduced from a new point of view, and have been put in a simplified form for computation. Two direct methods for the measurement of inductance have been tried and found to agree with the values found by computation. These methods were, however, less satisfactory than direct computation. The time constants of a number of shunts at the Bureau of Standards and Harvard University have been determined to within one or two tenths of a microsecond. These may now be used as standards for the calibration of other shunts. The inductance and susceptibility to stray fields of a number of commercial types of shunt have been measured. In some cases a change of resistance in the presence of the external field has been detected.

Washington, December 30, I9I 5. 


\section{NOTATION}

$A, B, C, D \ldots \ldots$. Differences in mutual inductance.

$a, a_{1}, a_{2} \ldots \ldots \ldots$. Radii of wires or tubes.

$b, c \ldots \ldots \ldots \ldots$. Thickness of strips.

$d, d_{11} \ldots \ldots \ldots \ldots \ldots$ Distance c. to c. of wires, strips, or filaments.

$D_{11}, D_{12} \ldots \ldots \ldots \ldots$. Geometric mean distances.

E................ Maximum voltage.

e............... Instantaneous voltage.

$f \ldots \ldots \ldots \ldots \ldots$. Frequency.

$g \ldots \ldots \ldots \ldots \ldots$ Thickness of insulation between strips.

$H \ldots \ldots \ldots \ldots \ldots$ Magnetic field intensity.

$I_{m}, i \ldots \ldots \ldots \ldots$ Maximum and instantaneous currents.

$i_{1}, i_{\mathrm{e}} \ldots \ldots \ldots \ldots$. Instantaneous current densities.

$j \ldots \ldots \ldots \ldots \ldots \sqrt{-\mathrm{r}}$.

$K \ldots \ldots \ldots \ldots$ Undetermined constant.

L.............. Inductance.

$l \ldots \ldots \ldots \ldots \ldots$ Length of circuits.

$M, m \ldots \ldots \ldots \ldots$ Mutual inductances.

n.............. Pitch of winding.

$P \ldots \ldots \ldots \ldots$ Ratio of current transformer.

$R$.............. Resistance.

$r$................. Radius of coil.

$s, t, u \ldots \ldots \ldots \ldots$ Shape ratios of tubes.

$S_{1}, S_{2} \ldots \ldots \ldots$ Cross-sectional areas of conductors.

t............... Time.

$T$. . . . . . . . Time constant.

w..............Width of strip.

$x \ldots \ldots \ldots \ldots$ Distance from axis to filament considered.

$y \ldots \ldots \ldots \ldots$ Distance from axis to equivalent filament.

$\alpha, \beta, \gamma, \delta, \eta, \kappa, \lambda, s \ldots$. Shape ratios of strip shunts.

$\theta$............... Phase angle of shunt.

r............... Apparent phase angle of transformer.

ע............... Correction term.

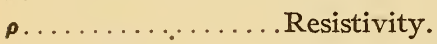

$\tau$.............. Phase defect of a mutual inductance.

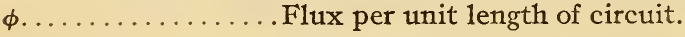

$\varphi \ldots \ldots \ldots \ldots$ True angle of transformer.

$\omega \ldots \ldots \ldots \ldots \ldots .2 \pi \times$ frequency. 